\title{
Selecting Personnel with the Weighted Cross-Entropy TOPSIS of Hesitant Picture Fuzzy Linguistic Sets
}

\author{
Xiao-Hui Wu $\mathbb{D}^{1,2,3}$ Lin Yang, $^{2}$ and Jie Qian ${ }^{2}$ \\ ${ }^{1}$ School of Economics and Management, Yancheng Institute of Technology, Yancheng, Jiangsu 224051, China \\ ${ }^{2}$ Institute of Big Data Intelligent Decision-Making, Hubei University of Automotive Technology, Shiyan 442002, China \\ ${ }^{3}$ School of Business, Central South University, Changsha 410083, China
}

Correspondence should be addressed to Xiao-Hui Wu; 254700361@qq.com

Received 4 July 2021; Revised 8 October 2021; Accepted 9 October 2021; Published 20 November 2021

Academic Editor: Jun Ye

Copyright (c) 2021 Xiao-Hui Wu et al. This is an open access article distributed under the Creative Commons Attribution License, which permits unrestricted use, distribution, and reproduction in any medium, provided the original work is properly cited.

Personnel selection is a key important role for the human resource department of organization, and hesitant picture fuzzy linguistic sets (HPFLSs) elaborated the advantages of both hesitant linguistic set and picture fuzzy set, which is more flexible and effective to solve the decision-making problems of personnel selection than other extension of fuzzy linguistic sets (FLSs). Crossentropy, as effective measurement tools, is wildly used under fuzzy multicriteria decision-making (FMCDM) environment; thus, in order to elaborate the advantages of both cross-entropy and HPFLSs under FMCDM environment, the cross-entropy definition of HPFLSs is firstly given in this paper. Meanwhile, several novel cross-entropy measures between two HPFLSs are introduced, and their related properties are proved. Then, an approach based on the weighted cross-entropy measures and TOPSIS under hesitant picture fuzzy linguistic environment is proposed. Finally, the proposed method is applied to the real personnel's selection, and the ranking results show that the proposed methods are practical and effective.

\section{Introduction}

Selecting personnel is the key role faced by the human resource (HR) department in organization, and multicriteria decision-making (MCDM) methods, as one of the effective tools, can be used to solve these problems. Due to the existing vague and uncertainty evaluation information in the process of personnel selection, fuzzy set-based MCDM approaches [1-7] were proposed by many researchers. Sang et al. [2] proposed the fuzzy TOPSIS-based method to solve personnel selection for knowledge-intensive enterprise. Baležentis et al. [4] conducted personnel selection approach based on computing with words and fuzzy MULTIMOORA, and Lin [5] proposed the personnel selection method based on the analytic network process and fuzzy data envelopment analysis. However, FSs-based approaches can only express evaluation information with membership degree and cannot express evaluation information with nonmembership degree. Thus, intuitionistic fuzzy set (IFS) based MCDM approaches [8-10] were put forward to solve personnel selection problems. Zhang and Liu [8] proposed GRA-based intuitionistic fuzzy (IF) multicriteria group decision-making method for personnel selection. Boran et al. [9] presented the IFS-based method for personnel selection. Kilic et al. [10] proposed an integrated decision analysis methodology based on IF-DEMATEL and IF-ELECTRE for personnel selection. Considering the real personnel selection situation, human resource managers can be hesitant to express preference while evaluating personnel, so Yu et al. [11] conducted hesitant fuzzy sets (HFSs) based MCDM approaches to aid managers of the human resource (HR) department for selecting excellent personnel.

As so far, many extensive FS-based MCDM approaches [2-11] have been conducted and helped managers of HR department to well solve decision-making problems of personnel selection. In some real circumstance, personnel evaluation may involve indeterminate and inconsistent information; thus, several neutrosophic-based MCDM methods [12-15] for personnel selection have been proposed by many researchers, in order to elaborate the advantages of 
TODIM and multivalued neutrosophic set. $\mathrm{Pu}$ et al. [12] conducted a projection-based TODIM method with a multivalued neutrosophic set for personnel selection. Nabeeh et al. [13] proposed an integrated neutrosophicTOPSIS approach for personnel selection, considering crossentropy as the major measurement for MCDM approaches. $\mathrm{Wu}$ et al. $[14,15]$ proposed two methods for middle-level manager selections with cross-entropy of probability hesitant interval neutrosophic set and multivalued neutrosophic sets.

Although these above proposed MCDM approaches for personnel selection can demonstrate the effectiveness under practical environment, these approaches cannot be effectively utilized to solve personnel selection decision-making problems with different attitude information from decisionmakers or experts. For such kind of personnel selection situations, it is required to present new approaches.

Picture fuzzy set (PFS) [16] was firstly proposed by Cường and used to describe different attitude information by many researchers. Until now, many MCDM methods based on the picture fuzzy set [17-22] were proposed. Tian et al. [17, 20, 21] proposed PFS-based MCDM methods, which are used for tourism attraction recommendation and tourism environmental impact assessment. Wang et al. [19] proposed a bounded rationality behavioral decision support model with picture fuzzy information, which is applied to select the different hotel among various types of travelers. However, in the real personnel selection decision-making environment, the interviewers might be hesitant to give the evaluation among several linguistic terms, such as "outstanding excellent," "excellent," and "merit" linguistic terms. Meanwhile, different interviewer has different attitude for the evaluation value. For example, one listed company carried out personnel selection interview; there are several job applicants for the interview by company interviewers; in the interview process, ten interviewers are required to give the evaluation score for the job applicants. Three interviewers are unsatisfied with all job applicants and refused to give any evaluation value; seven interviewers opposed to give the applicant with the score "outstanding excellent," but they support to give the evaluation value with the score "excellent." Due to such kinds of decision-making issues, PFS cannot be used to well describe the evaluation information. In order to avoid the information loss, the proposed new extensive PFS-based methods are required.

HPFLSs [23], as the extensive set of PFS, elaborated the advantages of both hesitant linguistic set and picture fuzzy set, which are more suitable for the practical hesitant decision-making problem together with different attitude information. As mentioned, the above example of job applicant interview, if the evaluation linguistic terms set is $\left\{s_{5}=\right.$ outstading excellent; $s_{4}=$ excellent; $s_{3}=$ merit; $\quad s_{2}=$ average; $s_{1}=$ pass; $s_{0}=$ failure $\}$, then the evaluation value with different attitude information provided by the interviewers can be collected as a set of $\left\langle\left\{\left(s_{4}\right),(0.7,0,0)\right\},\left\{\left(s_{5}\right),(0,0,0.7)\right\}\right\rangle$, and it is called as HPFLSs.

In the following, the contributions of this paper are presented as follows:
(1) The cross-entropy measure definition of hesitant picture fuzzy linguistic number is given, and its properties needed to be satisfied are listed. Meanwhile, the comparison rules of hesitant fuzzy linguistic set are defined.

(2) Based on the cross-entropy definition of hesitant picture fuzzy linguistic number, several formulas of cross-entropy of hesitant fuzzy linguistic number are constructed, and the relative properties are proven as well.

(3) Considering the real-life application, the weight of criteria cannot be provided in time due to some pressures or reasons. The method is presented to obtain the unknown weight of criteria.

(4) In accordance with the proposed cross-entropy formula, the MCDM approaches based on crossentropy of hesitant picture fuzzy linguistic numbers and TOPSIS are constructed.

The rest of the paper is organized as follows: some definitions related to picture fuzzy linguistic set and HPFLSs are introduced in Section 2. In Section 3, several novel formulas of cross-entropy measure under HPFLSs environment are proposed, and the related mathematics-induced proofs are shown. By utilizing the proposed crossentropy measure, in Section 4, an approach of the detailed decision-making steps for solving the MCDM problem under HPFLSs environment with completely unknown criteria weight is presented. Furthermore, a practical application on the MCDM problem of personnel's selection is conducted to demonstrate the effectiveness of the proposed method in Section 5. Finally, conclusion and further direction is drawn in Section 6.

\section{Preliminaries}

In this section, the definitions of cross-entropy measurement, the PFS, 2TFLSs, 2TLPFSs, HPFLSs, and cross-entropy of FS and PFS are presented to lay the groundwork for later analysis.

\subsection{Definitions of PFS, 2TFLSs, 2TLPFSs, and HPFLSs}

Definition 1 (see [16]). Let $X$ be a universe space. A PFS is defined as

$$
A=\left\{\left(x, \mu_{A}(x), \eta_{A}(x), v_{A}(x)\right) \mid x \in X\right\},
$$

where $\mu_{A}(x) \in[0,1]$ is called the degree of positive membership of $x$ in $A, \eta_{A}(x) \in[0,1]$ is called the degree of neutral membership of $x$ in $A, v_{A}(x) \in[0,1]$ is called the degree of negative membership of $x$ in $A$, and $\mu_{A}(x), \eta_{A}(x)$, $v_{A}(x)$ satisfy the condition $0 \leq \mu_{A}(x)+\eta_{A}(x)+v_{A}(x) \leq 1$. Moreover, $\pi_{A}(x)=1-\left(\mu_{A}(x)+\eta_{A}(x)+v_{A}(x)\right)$ can be called the degree of refusal.

Let $S=\left\{s_{j} \mid j=0,1,2, \ldots, g\right\}$ be a linguistic term set. Symbolic method aggregation linguistic information obtains a value $\beta \in[0, g]$, and if $\beta \notin[0, g]$, then an approximation function $\left(\operatorname{app}_{2}(\cdot)\right)$ is used to express the index result of $S$. 
Definition 2 (see [24]). Let $\beta$ be the aggregation result of the indices of a set of labels assessed in a linguistic term set $S$, i.e., the results of symbolic aggregation operation, $\beta \in[1, t]$ be the $t$ cardinality of $S$. Let $i=\operatorname{round}(\beta)$ and $\alpha=\beta-i$ be two values, such that $i \in[1, t]$ and $\alpha \in[-0.5,0.5)$; then, $\alpha$ is called the symbolic translation.

For convenience to collect the data in the selection of the proxy advisor firm problems, Nie et al. [25] defined the concept of 2-tuple linguistic picture fuzzy sets (2TLPFSs).

Definition 3. Let $S=\left\{s_{j} \mid j=0,1,2, \ldots, g\right\}$ be a linguistic set and $\bar{S}=\left\{\left(s_{q}, \alpha_{q}\right) \mid s_{q} \in S, \alpha_{q} \in[0.5,0.5]\right\}$ be a linguistic 2tuple fuzzy set. Suppose that $\left(s_{u}, \alpha_{u}\right), \quad\left(s_{\eta}, \alpha_{\eta}\right)$, and $\left(s_{\nu}, \alpha_{\nu}\right) \in \bar{S} ; \quad$ if $\quad 0 \leq \mu+\eta+\nu \leq g$, then $\bar{r}=\left[\left(s_{u}, \alpha_{u}\right),\left(s_{\eta}, \alpha_{\eta}\right),\left(s_{\nu}, \alpha_{v}\right)\right]$ is called 2TLPFSs, and $\left(s_{u}, \alpha_{u}\right),\left(s_{\eta}, \alpha_{\eta}\right)$, and $\left(s_{\gamma}, \alpha_{\nu}\right)$ could be called the positive membership degree, neutral membership degree, and negative membership degree of $\bar{r}$, respectively.

Considering the real-life decision-making environment, decision-makers might be hesitant to provide the evaluation information with different attitude; thus, Yang et al. [23] defined hesitant picture fuzzy linguistic sets (HPFLSs).

Definition 4. Let $S=\left\{s_{J} \mid J=0,1,2, \ldots, m\right\}$ be an LTS. An HPFLSs is defined as

$$
H_{s}=\left\{\left\langle\left(s_{i}^{k}\right),\left(\mu_{i}^{k}, \eta_{i}^{k}, v_{i}^{k}\right)\right\rangle \mid i \in(0,1,2, \ldots, m) ; k=1,2, \ldots, \alpha\right\},
$$

where $\mu_{i}^{k} \in[0,1], \quad \eta_{i}^{k} \in[0,1], \quad \nu_{i}^{k} \in[0,1], \quad$ and $0 \leq \mu_{i}^{k}+$ $\eta_{i}^{k}+\nu_{i}^{k} \leq 1 . \mu_{i}^{k}, \eta_{i}^{k}$, and $\nu_{i}^{k}$ represent the positive membership, indeterminacy membership, and negative membership of the linguistic term $s_{i}^{k}$, respectively; $\pi_{i}^{k}=1-\left(\mu_{i}^{k}+\eta_{i}^{k}+\nu_{i}^{k}\right)$ is the refusal membership of the linguistic term $s_{i}^{k}$. And, its complementary set is $H_{s}^{c}=\left\{\left\langle\left(s_{i}^{k}\right),\left(\nu_{i}^{k}, \eta_{i}^{k}, \mu_{i}^{k}\right)\right\rangle \mid i \in\right.$ $(0,1,2, \ldots, m) ; k=1,2, \ldots, \alpha\}$.

2.2. Cross-Entropy Measure of FS and PFS. The cross-entropy measure was introduced by Kullback [26], and its definition is shown as follows.

Definition 5 (see [26]). Let $P=\left\{p_{1}, p_{2}, \ldots, p_{n}\right\}$ and $Q=\left\{q_{1}, q_{2}, \ldots, q_{n}\right\}$ be two given probability distributions, where $p_{i} \geq 0 \quad \sum_{i=1}^{n} p_{i}=1 \quad$ and $q_{i} \geq 0 \quad \sum_{i=1}^{n} q_{i}=1$ for $i=(1,2, \ldots, n)$. The cross-entropy measure of $P$ to $Q$ is defined as

$$
H(P, Q)=\sum_{i=1}^{n} P_{i} * \ln \frac{p_{i}}{q_{i}}
$$

Based on Kullback's entropy definition, Shang and Jiang [27] proposed the cross-entropy measure between two fuzzy sets.

Definition 6. Assume that $A=\left\{A\left(x_{1}\right), A\left(x_{2}\right), \ldots, A\left(x_{n}\right)\right\}$ and $B=\left\{B\left(x_{1}\right), B\left(x_{2}\right), \ldots, B\left(x_{n}\right)\right\}$ are two fuzzy sets in the universe of discourse $X=\left\{x_{1}, x_{2}, \ldots, x_{n}\right\}$. The fuzzy crossentropy of $A$ from $B$ is defined as follows:

$$
H(A, B)=\sum_{i=1}^{n}\left(A\left(x_{i}\right) \log _{2}^{A\left(x_{i}\right) /(1 / 2)\left(A\left(x_{i}\right)+B\left(x_{i}\right)\right)}+\left(1-A\left(x_{i}\right)\right) \log _{2}^{\left(1-A\left(x_{i}\right)\right) /\left(1-(1 / 2)\left(A\left(x_{i}\right)+B\left(x_{i}\right)\right)\right)}\right)
$$

which indicates the degree of discrimination of $A$ from $B$.

However, $H(A, B)$ is not symmetric with respect to its arguments. Shang and Jiang [27] proposed a symmetric discrimination information measure: $I(A, B)=H(A, B)+H(B, A)$.
Wei [28] extended cross-entropy of fuzzy sets to picture fuzzy sets and conducted the cross-entropy of the picture fuzzy set.

Definition 7. Let $A$ and $B$ be two picture fuzzy sets, then the cross entropy measure of $A$ and $B$ are obtained as follows:

$$
\begin{aligned}
C(A, B)= & \sum_{i=1}^{n}\left(\mu_{A}\left(x_{i}\right) \ln \frac{\mu_{A}\left(x_{i}\right)}{(1 / 2)\left(\mu_{A}\left(x_{i}\right)+\mu_{B}\left(x_{i}\right)\right)}+\left(1-\mu_{A}\left(x_{i}\right)\right) \ln \frac{1-\mu_{A}\left(x_{i}\right)}{1-(1 / 2)\left(\mu_{A}\left(x_{i}\right)+\mu_{B}\left(x_{i}\right)\right)}\right) \\
& +\sum_{i=1}^{n}\left(\eta_{A}\left(x_{i}\right) \ln \frac{\eta_{A}\left(x_{i}\right)}{(1 / 2)\left(\eta_{A}\left(x_{i}\right)+\eta_{B}\left(x_{i}\right)\right)}+\left(1-\eta_{A}\left(x_{i}\right)\right) \ln \frac{1-\eta_{A}\left(x_{i}\right)}{1-(1 / 2)\left(\eta_{A}\left(x_{i}\right)+\eta_{B}\left(x_{i}\right)\right)}\right) \\
& +\sum_{i=1}^{n}\left(v_{A}\left(x_{i}\right) \ln \frac{v_{A}\left(x_{i}\right)}{(1 / 2)\left(v_{A}\left(x_{i}\right)+v_{B}\left(x_{i}\right)\right)}+\left(1-v_{A}\left(x_{i}\right)\right) \ln \frac{1-v_{A}\left(x_{i}\right)}{1-(1 / 2)\left(v_{A}\left(x_{i}\right)+v_{B}\left(x_{i}\right)\right)}\right) .
\end{aligned}
$$


Due to $C(A, B)$ being nonsymmetric, a symmetric discrimination cross-entropy measure is presented as $I(A, B)=C(A, B)+C(B, A)$.

\section{The Definition and Formula of HPFLS Cross-Entropy Measure}

In this section, the cross-entropy definition of HPFLSs is given. Considering that the previous cross-entropy formula [28] ignored the influence of refusal membership and different decision maker (DM) might have different support preference for different memberships, several formulas of HPFLSs, which overcome the limitations of previous crossentropy, are constructed.

Definition 8 . Let $H_{s_{1}}=\left\{\left\langle\left(s_{i_{1}}^{k_{1}}\right),\left(\mu_{i_{1}}^{k_{1}}, \eta_{i_{1}}^{k_{1}}, v_{i_{1}}^{k_{1}}\right)\right\rangle \mid i_{1} \in\{0,1,2\right.$, $\left.\ldots, m\} ; k_{1}=1,2, \ldots, \alpha_{1}\right\}$ and $H_{s_{2}}=\left\{\left\langle\left(s_{i_{2}}^{k_{2}}\right),\left(\mu_{i_{2}}^{k_{2}}, \eta_{i_{2}}^{k_{2}}, v_{i_{2}}^{k_{2}}\right)\right\rangle\right.$ $\left.\mid i_{2} \in\{0,1,2, \ldots, m\} ; k_{2}=1,2, \ldots, \alpha_{2}\right\}$ be two HPFLSs. $H_{s_{1}}$ is greater than or equal to $H_{s_{2}}$, denoted by $H_{s_{1}} \geq H_{s_{2}}$, if and only if $\mu_{1} \geq \mu_{2}, \eta_{1} \leq \eta_{2}, v_{1} \leq v_{2}$, where

$$
\begin{aligned}
& \mu_{1}=\sqrt[\alpha 1]{\sum_{k_{1}=1}^{\alpha_{1}} \frac{\left(\mu_{k_{1}} \times i_{k_{1}}\right)^{\alpha_{1}}}{\alpha_{1}}} \\
& \mu_{2}=\sqrt[\alpha 2]{\sum_{k_{2}=1}^{\alpha_{2}} \frac{\left(\mu_{k_{2}} \times i_{k_{2}}\right)^{\alpha_{2}}}{\alpha_{2}}} \\
& \eta_{1}=\sqrt[\alpha_{1}]{\sum_{k_{1}=1}^{\alpha_{1}} \frac{\left(\eta_{k_{1}} \times i_{k_{1}}\right)^{\alpha_{1}}}{\alpha_{1}}} \\
& \eta_{2}=\sqrt[\alpha_{2}]{\sum_{k_{2}=1}^{\alpha_{2}} \frac{\left(\eta_{k_{2}} \times i_{k_{2}}\right)^{\alpha_{2}}}{\alpha_{2}}} \\
& v_{1}=\sqrt[\alpha_{1}]{\sum_{k_{1}=1}^{\alpha_{1}} \frac{\left(v_{k_{1}} \times i_{k_{1}}\right)^{\alpha_{1}}}{\alpha_{1}}}
\end{aligned}
$$

$$
v_{2}=\sqrt[\alpha 2]{\sum_{k_{2}=1}^{\alpha_{2}} \frac{\left(v_{k_{2}} \times i_{k_{2}}\right)^{\alpha_{1}}}{\alpha_{2}}}
$$

$i_{k_{j}}=\left(1+\exp \left(\left(i_{j}-m / 2\right) \times \theta\right)\right)^{-n}(j=1,2)$ and $i_{j}$ are the subscript values of the linguistic assessment term $s_{i_{j}}^{k_{j}}$ and $n>0, \quad \theta>0, \quad \mu_{k_{j}}=\left(\mu_{i_{j}}^{k_{j}}+1-\eta_{i_{j}}^{k_{j}}-v_{i_{j}}^{k_{j}}\right) / 2, \quad \eta_{k_{j}}=\left(\eta_{i_{j}}^{k_{j}}+1-\right.$ $\left.\mu_{i_{j}}^{k_{j}}-v_{i_{j}}^{k_{j}}\right) / 2$, and $v_{k_{j}}=\left(v_{i_{j}}^{k_{j}}+1-\eta_{i_{j}}^{k_{j}}-\mu_{i_{j}}^{k_{j}}\right) / 2, j=1,2$.

Definition 9. Assume that $H_{s_{1}}=\left\{\left\langle\left(s_{i_{1}}^{k_{1}}\right),\left(\mu_{i_{1}}^{k_{1}}, \eta_{i_{1}}^{k_{1}}, v_{i_{1}}^{k_{1}}\right)\right\rangle \mid i_{1}\right.$ $\left.\in\{0,1,2, \ldots, m\} ; k_{1}=1,2, \ldots, \alpha_{1}\right\}$ and $H_{s_{2}}=\left\{\left\langle\left(s_{i_{2}}^{k_{2}}\right),\left(\mu_{i_{2}}^{k_{2}}\right.\right.\right.$, $\left.\left.\left.\eta_{i_{2}}^{k_{2}}, v_{i_{2}}^{k_{2}}\right)\right\rangle \mid i_{2} \in\{0,1,2, \ldots, m\} ; k_{2}=1,2, \ldots, \alpha_{2}\right\}$ are two HPFLS, CE* ${ }^{*}$ HPFLS $\times$ HPFLS $\longrightarrow R^{+}$. The cross-entropy $C E^{*}\left(H_{s_{1}}, H_{s_{2}}\right)$ of $H_{s_{1}}$ and $H_{s_{2}}$ should satisfy the following conditions:

(1) $C E^{*}\left(H_{s_{1}}, H_{s_{2}}\right)=C E^{*}\left(H_{s_{2}}, H_{s_{1}}\right), \forall H_{s_{1}}, H_{s_{2}} \in$ HPFLS

(2) $C E^{*}\left(H_{s_{1}}^{c}, H_{s_{2}}^{c}\right)=C E^{*}\left(H_{s_{1}}, H_{s_{2}}\right), \forall H_{s_{1}}, H_{s_{2}} \in$ HPFLS

(3) $C E^{*}\left(H_{s_{1}}, H_{s_{2}}\right) \geq 0, \forall H_{s_{1}}, H_{s_{2}} \in \mathrm{HPFLS}$, if $H_{s_{1}}=H_{s_{2}}$, then $C E^{*}\left(H_{s_{1}}, H_{s_{2}}\right)=0$

(4) $C E^{*}\left(H_{s_{1}}, H_{s_{3}}\right) \geq C E^{*}\left(H_{s_{1}}, H_{s_{2}}\right)$ and $C E^{*}\left(H_{s_{1}}, H_{s_{3}}\right)$ $\geq C E^{*}\left(H_{s_{2}}, H_{s_{3}}\right), \forall H_{s_{1}}, H_{s_{2}}, \quad H_{s_{3}} \in$ HPFLS and if $H_{s_{1}} \geq H_{s_{2}} \geq H_{s_{3}}$

Based on the above definitions, the cross-entropy formulas of HPFLSs are obtained as follows:

$$
\begin{aligned}
& C E_{1}\left(H_{s_{1}}, H_{s_{2}}\right)=\rho \times \sin \left(\sqrt[\alpha_{1}]{\sum_{k_{1}=1}^{\alpha_{1}} \frac{\left(\mu_{k_{1}} * i_{k_{1}}\right)^{\alpha_{1}}}{\alpha_{1}}}\right) \times \sin \left(\sqrt[\alpha_{1}]{\sum_{k_{1}=1}^{\alpha_{1}} \frac{\left(\mu_{k_{1}} * i_{k_{1}}\right)^{\alpha_{1}}}{\alpha_{1}}}-\sqrt[\alpha_{2}]{\sum_{k_{2}=1}^{\alpha_{2}} \frac{\left(\mu_{k_{2}} * i_{k_{2}}\right)^{\alpha_{2}}}{\alpha_{2}}}\right) \\
& +\varphi \times \sin \left(\sqrt[\alpha 1]{\sum_{k_{1}=1}^{\alpha_{1}} \frac{\left(\eta_{k_{1}} * i_{k_{1}}\right)^{\alpha_{1}}}{\alpha_{1}}}\right) \times \sin \left(\sqrt[\alpha 1]{\sum_{k_{1}=1}^{\alpha_{1}} \frac{\left(\eta_{h_{1}} * i_{k_{1}}\right)^{\alpha_{1}}}{\alpha_{1}}}-\sqrt[\alpha 2]{\sum_{k_{2}=1}^{\alpha_{2}} \frac{\left(\eta_{k_{2}} * i_{k_{2}}\right)^{\alpha_{2}}}{\alpha_{2}}}\right) \\
& +\delta \times \sin \left(\sqrt[\alpha 1]{\sum_{k_{1}=1}^{\alpha_{1}} \frac{\left(v_{k_{1}} * i_{k_{1}}\right)^{\alpha_{1}}}{\alpha_{1}}}\right) \times \sin \left(\sqrt[\alpha 1]{\sum_{k_{1}=1}^{\alpha_{1}} \frac{\left(v_{k_{1}} * i_{k_{1}}\right)^{\alpha_{1}}}{\alpha_{1}}}-\sqrt[\alpha 2]{\sum_{k_{2}=1}^{\alpha_{2}} \frac{\left(v_{k_{2}} * i_{k_{2}}\right)^{\alpha_{2}}}{\alpha_{2}}}\right) .
\end{aligned}
$$


Similarly, other three cross-entropy formulas of HPFLSs are obtained as follows:

$$
\begin{aligned}
& C E_{2}\left(H_{s_{1}}, H_{s_{2}}\right)=\rho \times \tan \left(\sqrt[\alpha 1]{\sum_{k_{1}=1}^{\alpha_{1}} \frac{\left(\mu_{k_{1}} \times i_{k_{1}}\right)^{\alpha_{1}}}{\alpha_{1}}}\right) \times \tan \left(\sqrt[\alpha_{1}]{\sum_{k_{1}=1}^{\alpha_{1}} \frac{\left(\mu_{k_{1}} \times i_{k_{1}}\right)^{\alpha_{1}}}{\alpha_{1}}}-\sqrt[\alpha_{2}]{\sum_{k_{2}=1}^{\alpha_{2}} \frac{\left(\mu_{k_{2}} \times i_{k_{2}}\right)^{\alpha_{2}}}{\alpha_{2}}}\right) \\
& +\varphi \times \tan \left(\sqrt[\alpha_{1}]{\sum_{k_{1}=1}^{\alpha_{1}} \frac{\left(\eta_{k_{1}} \times i_{k_{1}}\right)^{\alpha_{1}}}{\alpha_{1}}}\right) \times \tan \left(\sqrt[\alpha_{1}]{\sum_{k_{1}=1}^{\alpha_{1}} \frac{\left(\eta_{k_{1}} \times i_{k_{1}}\right)^{\alpha_{1}}}{\alpha_{1}}}-\sqrt[\alpha_{2}]{\sum_{k_{2}=1}^{\alpha_{2}} \frac{\left(\eta_{k_{2}} \times i_{k_{2}}\right)^{\alpha_{2}}}{\alpha_{2}}}\right) \\
& +\delta \times \tan \left(\sqrt[\alpha 1]{\sum_{k_{1}=1}^{\alpha_{1}} \frac{\left(v_{k_{1}} \times i_{k_{1}}\right)^{\alpha_{1}}}{\alpha_{1}}}\right) \times \tan \left(\sqrt[\alpha 1]{\sum_{k_{1}=1}^{\alpha_{1}} \frac{\left(v_{k_{1}} \times i_{k_{1}}\right)^{\alpha_{1}}}{\alpha_{1}}}-\sqrt[\alpha_{2}]{\sum_{k_{2}=1}^{\alpha_{2}} \frac{\left(v_{k_{2}} \times i_{k_{2}}\right)^{\alpha_{2}}}{\alpha_{2}}}\right) \\
& C E_{3}\left(H_{s_{1}}, H_{s_{2}}\right)=\rho \times\left(\sqrt[\sum_{k_{1}=1}^{\alpha_{1}} \frac{\left(\mu_{k_{1}} \times i_{k_{1}}\right)^{\alpha_{1}}}{\alpha_{1}}]{1}\right) \times \ln \frac{2 \times\left(\sqrt[\alpha 1]{\sum_{k_{1}=1}^{\alpha_{1}}\left(\mu_{k_{1}} \times i_{k_{1}}\right)^{\alpha_{1}} / \alpha_{1}}+1\right)}{2+\sqrt[\alpha 1]{\sum_{k_{1}=1}^{\alpha_{1}}\left(\mu_{k_{1}} \times i_{k_{1}}\right)^{\alpha_{1}} / \alpha_{1}}+\sqrt[\alpha 2]{\sum_{k_{2}=1}^{\alpha_{2}}\left(\mu_{k_{2}} \times i_{k_{2}}\right)^{\alpha_{2}} / \alpha_{2}}} \\
& +\varphi \times\left(\sqrt[\alpha 1]{\sum_{k_{1}=1}^{\alpha_{1}} \frac{\left(\eta_{k_{1}} \times i_{k_{1}}\right)^{\alpha_{1}}}{\alpha_{1}}}+1\right) \times \ln \frac{2 \times\left(\sqrt[\alpha 1]{\sum_{k_{1}=1}^{\alpha_{1}}\left(\eta_{k_{1}} \times i_{k_{1}}\right)^{\alpha_{1}} / \alpha_{1}}+1\right)}{2+\sqrt[\alpha 1]{\sum_{k_{1}=1}^{\alpha_{1}}\left(\eta_{k_{1}} \times i_{k_{1}}\right)^{\alpha_{1}} / \alpha_{1}}+\sqrt[\alpha 2]{\sum_{k_{2}=1}^{\alpha_{2}}\left(\eta_{k_{2}} \times i_{k_{2}}\right)^{\alpha_{2}} / \alpha_{2}}} \\
& +\delta \times\left(\sqrt[\alpha 1]{\sum_{k_{1}=1}^{\alpha_{1}} \frac{\left(v_{k_{1}} \times i_{k_{1}}\right)^{\alpha_{1}}}{\alpha_{1}}}+1\right) \times \ln \frac{2 \times\left(\sqrt[\alpha 1]{\sum_{k_{1}=1}^{\alpha_{1}}\left(v_{k_{1}} \times i_{k_{1}}\right)^{\alpha_{1}} / \alpha_{1}}+1\right)}{2+\sqrt[\alpha 1]{\sum_{k_{1}=1}^{\alpha_{1}}\left(v_{k_{1}} \times i_{k_{1}}\right)^{\alpha_{1}} / \alpha_{1}}+\sqrt[\alpha 2]{\sum_{k_{2}=1}^{\alpha_{2}}\left(v_{k_{2}} \times i_{k_{2}}\right)^{\alpha_{2}} / \alpha_{2}}}, \\
& C E_{4}\left(H_{s_{1}}, H_{s_{2}}\right)=\rho \sqrt[\alpha 1]{\sum_{k_{1}=1}^{\alpha_{1}} \frac{\left(\mu_{k_{1}} * i_{k_{1}}\right)^{\alpha_{1}}}{\alpha_{1}}} \times\left(\exp \left(\sqrt[\alpha 1]{\sum_{k_{1}=1}^{\alpha_{1}} \frac{\left(\mu_{k_{1}} \times i_{k_{1}}\right)^{\alpha_{1}}}{\alpha_{1}}}-1\right)-\exp \left(\sqrt[\alpha_{2}]{\left.\sum_{k_{2}=1}^{\alpha_{2}} \frac{\left(\mu_{k_{2}} \times i_{k_{2}}\right)^{\alpha_{2}}}{\alpha_{2}}-1\right)}\right)\right.
\end{aligned}
$$

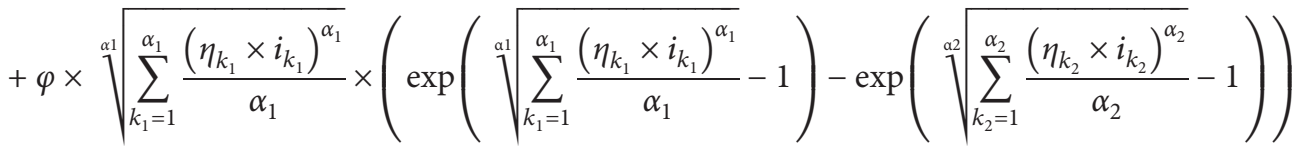

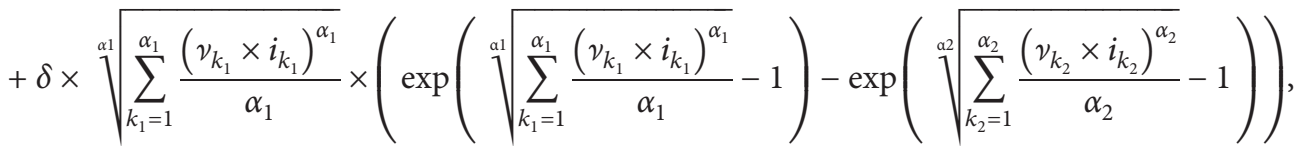

where $i_{k_{j}}=\left(1+\exp \left(\left(i_{j}-m / 2\right) \times \theta\right)\right)^{-n}(j=1,2)$ and $i_{j}$ are the subscript values of the linguistic assessment term $s_{i_{j}}^{k_{j}}$ and $n>0, \quad \theta>0, \quad \mu_{k_{j}}=\left(\mu_{i_{j}}^{k_{j}}+1-\eta_{i_{j}}^{k_{j}}-v_{i_{j}}^{k_{j}}\right) / 2, \quad \eta_{k_{j}}=\left(\eta_{i_{j}}^{k_{j}}+1-\right.$ $\left.\mu_{i_{j}}^{k_{j}}-v_{i_{j}}^{k_{j}}\right) / 2, \quad v_{k_{j}}=\left(\left(v_{i_{j}}^{k_{j}}+1-\eta_{i_{j}}^{k_{j}}-\mu_{i_{j}}^{k_{j}}\right) / 2\right), j=1,2, \rho+\varphi+$ $\delta=1$, and normally $\rho=\varphi=\delta=(1 / 3)$.

However, considering that the formulas $C E_{k}\left(H_{s_{1}}, H_{s_{2}}\right)$ $(k=1,2,3)$ are asymmetric, the above formulas are modified as follows:

$$
\begin{gathered}
C E_{k}^{*}\left(H_{s_{1}}, H_{s_{2}}\right)=C E_{k}\left(H_{s_{1}}, H_{s_{2}}\right)+C E_{k}\left(H_{s_{2}}, H_{s_{1}}\right), \\
k=1,2,3 .
\end{gathered}
$$

Property 1. Let $H_{s_{1}}=\left\{\left\langle\left(s_{i_{1}}^{k_{1}}\right),\left(\mu_{i_{1}}^{k_{1}}, \eta_{i_{1}}^{k_{1}}, v_{i_{1}}^{k_{1}}\right)\right\rangle \mid i_{1} \in\{0,1,2, \ldots\right.$, $\left.m\} ; k_{1}=1,2, \ldots, \alpha_{1}\right\}$ and $H_{s_{2}}=\left\{\left\langle\left(s_{i_{2}}^{k_{2}}\right),\left(\mu_{i_{2}}^{k_{2}}, \eta_{i_{2}}^{k_{2}}, v_{i_{2}}^{k_{2}}\right)\right\rangle \mid i_{2} \in\right.$ $\left.\{0,1,2, \ldots, m\} ; k_{2}=1,2, \ldots, \alpha_{2}\right\}$ be two HPFLSs. The crossentropy measure $C E_{k}^{*}\left(H_{s_{1}}, H_{s_{2}}\right)$ is defined as the discrimination degree from $H_{s_{1}}$ to $H_{s_{2}}$, and then $C E_{k}^{*}\left(H_{s_{1}}, H_{s_{2}}\right)$ satisfies conditions (1)-(4) in Definitions 1 and 1 .

Proof. Since it is easily proven that conditions (7) and (8) hold, their proofs are omitted here. For condition (9), the detailed proof is shown as follows.

Given that $u_{1}=\sqrt[\alpha 1]{\sum_{k_{1}=1}^{\alpha_{1}}\left(\mu_{k_{1}} \times i_{k_{1}}\right)^{\alpha_{1}} / \alpha_{1}}$ and $u_{2}=$ $\sqrt[\alpha 2]{\sum_{k_{2}=1}^{\alpha_{2}}\left(\mu_{k_{2}} \times i_{k_{2}}\right)^{\alpha_{2} / \alpha_{2}}}$, it is obvious to prove that the following constructed functions hold: 


$$
\begin{aligned}
f_{1}\left(u_{1}, u_{2}\right) & =\sin \left(u_{1}\right) \times \sin \left(u_{1}-u_{2}\right)+\sin \left(u_{2}\right) \times \sin \left(u_{2}-u_{1}\right) \\
& =\sin \left(u_{1}\right) \times \sin \left(u_{1}-u_{2}\right)-\sin \left(u_{2}\right) \times \sin \left(u_{1}-u_{2}\right) \\
& =\left(\sin \left(u_{1}\right)-\sin \left(u_{2}\right)\right) \times \sin \left(u_{1}-u_{2}\right) \geq 0, \\
f_{2}\left(u_{1}, u_{2}\right) & =\tan \left(u_{1}\right) \times \tan \left(u_{1}-u_{2}\right)+\tan \left(u_{2}\right) \times \tan \left(u_{2}-u_{1}\right) \\
& =\tan \left(u_{1}\right) \times \tan \left(u_{1}-u_{2}\right)-\tan \left(u_{2}\right) \times \tan \left(u_{1}-u_{2}\right) \\
& =\left(\tan \left(u_{1}\right)-\tan \left(u_{2}\right)\right) \times \tan \left(u_{1}-u_{2}\right) \geq 0, \\
f_{3}\left(u_{1}, u_{2}\right) & =\left(\mu_{1}+1\right) \times \ln \frac{2 \times\left(\mu_{1}+1\right)}{2+\mu_{1}+\mu_{2}}+\left(\mu_{2}+1\right) \times \ln \frac{2 \times\left(\mu_{2}+1\right)}{2+\mu_{1}+\mu_{2}} \\
& =\left(\mu_{1}+1\right) \times \ln \frac{\left(\mu_{1}+1\right)}{\left(\mu_{1}+\mu_{2}+2\right) / 2}+\left(\mu_{2}+1\right) \times \ln \frac{\left(\mu_{2}+1\right)}{\left(2+\mu_{1}+\mu_{2}\right) / 2} \\
& =\left(\mu_{1}+1\right) \times \ln \frac{\left(\mu_{1}+1\right)+\left(\mu_{2}+1\right) \times \ln \left(\mu_{2}+1\right)}{-\left(2+\mu_{1}+\mu_{2}\right) \times \ln \frac{\left(2+\mu_{1}+\mu_{2}\right) / 2}{},}
\end{aligned}
$$

where $\mu \in[1,2], f(\mu)=\mu \ln \mu$ is a concave function [29]; thus, it satisfies $f\left(\mu_{1}+1\right)+f\left(\mu_{2}+1\right) / 2 \geq f\left(2+\mu_{1}+\mu_{2} / 2\right)$, so $\mu_{1}, \mu_{2} \in[0,1]$ and $f_{3}\left(u_{1}, u_{2}\right) \geq 0$ hold.

$$
\begin{aligned}
f_{4}\left(u_{1}, u_{2}\right)= & u_{1} \times\left(\exp \left(u_{1}-1\right)-\exp \left(u_{2}-1\right)\right) \\
& +u_{2} \times\left(\exp \left(u_{2}-1\right)-\exp \left(u_{1}-1\right)\right) \\
= & \left(u_{1}-u_{2}\right) \times\left(\exp \left(u_{1}-1\right)-\exp \left(u_{2}-1\right)\right) \geq 0 .
\end{aligned}
$$

Similarly, given that $\eta_{1}=\sqrt[\alpha 1]{\sum_{k_{1}=1}^{\alpha_{1}}\left(\eta_{k_{1}} \times i_{k_{1}}\right)^{\alpha_{1}} / \alpha_{1}}, \eta_{2}=$ $\sqrt[\alpha 2]{\sum_{k_{2}=1}^{\alpha_{2}}\left(\eta_{k_{2}} \times i_{k_{2}}\right)^{\alpha_{2} / \alpha_{2}}}, \quad \nu_{1}=\sqrt[\alpha 1]{\sum_{k_{1}=1}^{\alpha_{1}}\left(\nu_{k_{1}} \times i_{k_{1}}\right)^{\alpha_{1} / \alpha_{1}}}$ and $v_{2}=\sqrt[\alpha 2]{\sum_{k_{2}=1}^{\alpha_{2}}\left(\nu_{k_{2}} \times i_{k_{2}}\right)^{\alpha_{2}} / \alpha_{2}}$, then it can be proven that the following inequalities $f_{i}\left(\eta_{1}, \eta_{2}\right) \geq 0, f_{i}\left(\nu_{1}, v_{2}\right) \geq 0, \quad(i=$ $1,2,3,4)$ hold.
According to Definitions 8 and 9, the following function can be obtained.

$$
\begin{aligned}
C E_{1}^{*}\left(H_{s_{1}}, H_{s_{2}}\right) & =C E_{1}\left(H_{s_{1}}, H_{s_{2}}\right)+C E_{1}\left(H_{s_{2}}, H_{s_{1}}\right) \\
& =f_{1}\left(u_{1}, u_{2}\right)+f_{1}\left(\eta_{1}, \eta_{2}\right)+f_{1}\left(v_{1}, v_{2}\right) \geq 0 .
\end{aligned}
$$

Thus, the inequalities $C E_{2}^{*}\left(H_{s_{1}}, H_{s_{2}}\right) \geq 0, C E_{3}^{*}\left(H_{s_{1}}\right.$, $\left.H_{s_{2}}\right) \geq 0, C E_{4}^{*}\left(H_{s_{1}}, H_{s_{2}}\right) \geq 0$ can hold; next, the detailed proof of condition (10) is shown as follows:

Assume that $H_{s_{1}} \geq H_{s_{2}} \geq H_{s_{3}}$; in accordance with Definition 8 , the inequalities of $\mu_{1} \geq \mu_{2} \geq \mu_{3}, \eta_{1} \leq \eta_{2} \leq \eta_{3}$, and $v_{1} \leq v_{2} \leq v_{3}$ can be obtained. Then,

$$
\begin{aligned}
f_{1}\left(u_{1}, u_{2}\right)= & \left(\sin \left(u_{1}\right)-\sin \left(u_{2}\right)\right) \times \sin \left(u_{1}-u_{2}\right) \\
\leq & \left(\sin \left(u_{1}\right)-\sin \left(u_{3}\right)\right) \times \sin \left(u_{1}-u_{3}\right) \\
\leq & f_{1}\left(u_{1}, u_{3}\right) \\
f_{2}\left(u_{1}, u_{2}\right)= & \left(\tan \left(u_{1}\right)-\tan \left(u_{2}\right)\right) \times \tan \left(u_{1}-u_{2}\right) \\
\leq & \left(\tan \left(u_{1}\right)-\tan \left(u_{3}\right)\right) \times \tan \left(u_{1}-u_{3}\right) \\
\leq & f_{2}\left(u_{1}, u_{3}\right), \\
f_{3}\left(u_{1}, u_{2}\right)-f_{3}\left(u_{1}, u_{3}\right)= & \left(\mu_{2}+1\right) \times \ln \left(\mu_{2}+1\right)-\left(\mu_{3}+1\right) \times \ln \left(\mu_{3}+1\right)+\left(2+\mu_{1}+\mu_{3}\right) \times \ln \left(2+\mu_{1}+\mu_{3}\right) / 2-\left(2+\mu_{1}+\mu_{2}\right) \times \ln \left(2+\mu_{1}+\mu_{2}\right) / 2 \\
\leq & \left(\mu_{2}+1\right) \times \ln \left(\mu_{2}+1\right)-\left(\mu_{3}+1\right) \times \ln \left(\mu_{3}+1\right)+\left(\mu_{1}+\mu_{2}+2\right) \times \ln \left(\mu_{1}+\mu_{2}+2\right) / 2-\left(\mu_{1}+\mu_{2}+2\right) \times \ln \left(\mu_{1}+\mu_{2}+2\right) / 2 \\
\leq & 0, \\
f_{4}\left(u_{1}, u_{2}\right)-f_{4}\left(u_{1}, u_{3}\right)= & u_{1} \times\left(\exp \left(u_{1}-1\right)-\exp \left(u_{2}-1\right)\right)+u_{2} \times\left(\exp \left(u_{2}-1\right)-\exp \left(u_{1}-1\right)\right) \\
& -u_{1} \times\left(\exp \left(u_{1}-1\right)-\exp \left(u_{3}-1\right)\right)-u_{3} \times\left(\exp \left(u_{3}-1\right)-\exp \left(u_{1}-1\right)\right) \\
\leq & 0 .
\end{aligned}
$$


Similarly, $f_{i}\left(\eta_{1}, \eta_{2}\right) \leq f_{i}\left(\eta_{1}, \eta_{3}\right), f_{i}\left(\nu_{1}, v_{2}\right) \leq f_{i}\left(\nu_{1}, v_{3}\right)$ $(i=1,2,3,4)$ can be proven. Thus, the proof of condition (10) is completely done.

\section{The MCDM Approach with Weighted Cross- Entropy and TOPSIS of HPFLSs}

In this section, the method based on Hamming distance and Lagrangian function is proposed to solve the MCDM problems under HPFLSs environment with unknown criteria weight, and the detailed decision-making steps under HPFLSs environment are summarized. For this sake, the hierarchical frame of the proposed method is presented as shown in Figure 1.
4.1. The Method of Unknown Criteria Weight Determination. In the practical decision-making environment, due to some emergency situation, the weight of the criterion cannot be provided in time. Therefore, the method based on the maximum-minimum distance is proposed to calculate the unknown criteria weight. In general, the criterion with the bigger distance value among the alternative should be assigned with bigger weight and vice versa [30,31]. In this section, the Hamming distance is used to calculate the distance. For a specific criterion $a_{4}$, the distance $\left\langle\left\{\left(s_{5}\right),(0.5\right.\right.$, $\left.0.3,0.1)\},\left\{\left(s_{4}\right),(0.5,0,0)\right\}\right\rangle$ between one alternative and other alternatives can be obtained by the following calculation formula:

$$
\begin{aligned}
D_{c_{q}} & =\frac{1}{m-1} \sum_{t=1}^{m} \sum_{e=1, e \neq t}^{m}\left(\mid H_{s_{t q}}-H_{s_{e q}}\right) \\
& =\frac{1}{m-1} \sum_{t=1}^{m} \sum_{e=1, e \neq t}^{m}\left(\left|\sum_{k_{t q=1}}^{\alpha_{t q}} i_{t q}-\sum_{k_{e q}=1}^{\alpha_{e q}} i_{e q}\right|+\left|\sum_{t_{t q}=1}^{\alpha_{t q}} \mu_{i_{t q}}^{k_{t q}}-\sum_{k_{e q}=1}^{\alpha_{e q}} \mu_{i_{e q}}^{k_{e q}}\right|+\left|\sum_{k_{t q=1}}^{\alpha_{t q}} \eta_{i_{t q}}^{k_{t q}}-\sum_{k_{e q}=1}^{\alpha_{e q}} \eta_{i_{e q}}^{k_{e q}}\right|+\left|\sum_{k_{t q=1}}^{\alpha_{t q}} \nu_{i_{t q}}^{k_{t q}}-\sum_{k_{e q}=1}^{\alpha_{e q}} \nu_{i_{e q}}^{k_{e q}}\right|\right) .
\end{aligned}
$$

The larger the value of $c_{3}$ is, the more important the corresponding criterion is. Next, the following model is constructed to determine the weight value.

$$
(M) \begin{cases}\max & D_{c}(w)=\sum_{q=1}^{n} w_{q} D_{c_{q}} \\ \text { s.t. } & \sum_{q=1}^{n} w_{q}^{2}=1, \quad w_{q} \geq 0, q=1,2, \ldots, n .\end{cases}
$$

Then, the above model $(M)$ is solved by constructing the Lagrangian function.

$$
L(w, \varepsilon)=\sum_{q=1}^{n} w_{q} D_{c_{q}}+\frac{\varepsilon}{2}\left(\sum_{q=1}^{n} w_{q}^{2}-1\right) .
$$

In order to compute the partial derivatives of $\left\langle\left\{\left(s_{5}\right),(0.6\right.\right.$, $\left.0,0)\},\left\{\left(s_{4}\right),(0.2,0,0)\right\}\right\rangle$ and $\left\langle\left\{\left(s_{5}\right),(0.8,0.1,0)\right\},\left\{\left(s_{4}\right),(0.2\right.\right.$, $0.2,0)\}\rangle$, the following partial derivative equations are obtained.

$$
\left\{\begin{array}{l}
\frac{\partial\left(w_{q}, \varepsilon\right)}{w_{q}}=D_{c_{q}}+\varepsilon w_{q}=0, \\
\frac{\partial\left(w_{q}, \varepsilon\right)}{\varepsilon}=\sum_{q=1}^{n} w_{q}^{2}-1=0 .
\end{array}\right.
$$

By solving formula (19), the formula for determining the weight vector is obtained as follows:

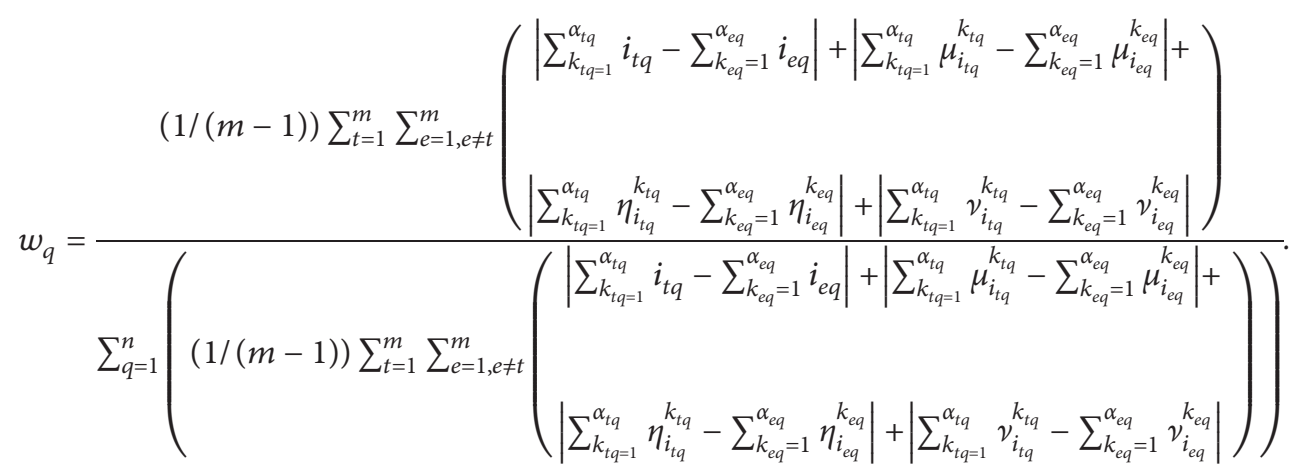




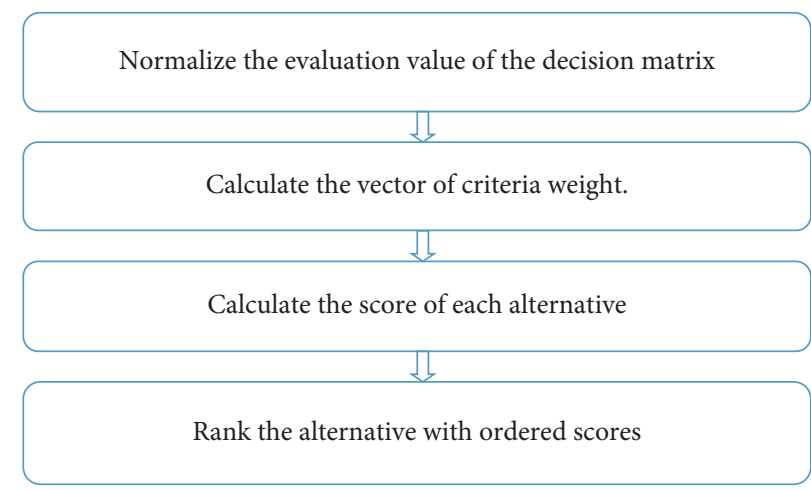

FIgURE 1: The hierarchical frame of the proposed cross-entropy-based HPFLSs MCGDM approach.

4.2. The Method with Weighted Cross-Entropy TOPSIS of HPFLSs. According to the above formula (11), weighted cross-entropy values from the alternative to positive ideal solution $H_{s}^{+}=\overbrace{\left\{\left\langle\left(s_{\max }\right),(1,0,0)\right\rangle, \ldots,\left\langle\left(s_{\max }\right),(1,0,0)\right\rangle\right\}}^{n}$ and the negative ideal solution $H_{s}^{-}=\overbrace{\left\{\left\langle\left(s_{\min }\right),(0,0,1)\right\rangle, \ldots,\left\langle\left(s_{\min }\right),(0,0,1)\right\rangle\right\}}^{n}$ can be obtained. The detailed process is shown as follows:

$$
\begin{aligned}
& C E_{1}^{*}\left(H_{s}, H_{s}^{+}\right)=C E_{1}\left(H_{s}, H_{s}^{+}\right)+C E_{1}\left(H_{s}^{+}, H_{s}\right) \\
& =\sum_{j=1}^{n} w_{j} \times\left(\rho \times \sin \left(\sqrt[a j]{\sum_{k_{j}=1}^{\alpha_{j}} \frac{\left(\mu_{k_{j}} \times i_{k_{j}}\right)^{\alpha_{j}}}{\alpha_{j}}}\right) \times \sin \left(\sqrt[a j]{\sum_{k_{j}=1}^{\alpha_{j}} \frac{\left(\mu_{k_{j}} \times i_{k_{j}}\right)^{\alpha_{j}}}{\alpha_{j}}}-i_{k}^{+}\right)+\varphi \times \sin \left(\sqrt[a_{j}]{\sum_{k_{j}=1}^{\alpha_{j}} \frac{\left(\eta_{k_{j}} \times i_{k_{j}}\right)^{\alpha_{j}}}{\alpha_{j}}}\right)\right. \\
& \times \sin \left(\sqrt[a_{j}]{\sum_{k_{j}=1}^{\alpha_{j}} \frac{\left(\eta_{k_{j}} \times i_{k_{j}}\right)^{\alpha_{j}}}{\alpha_{j}}}\right)+\delta \times \sin \left(\sqrt[a_{j}]{\sum_{k_{j}=1}^{\alpha_{j}} \frac{\left(v_{k_{j}} \times i_{k_{j}}\right)^{\alpha_{j}}}{\alpha_{j}}}\right) \times \sin \left(\sqrt[a_{j}]{\sum_{k_{j}=1}^{\alpha_{j}} \frac{\left(v_{k_{j}} \times i_{k_{j}}\right)^{\alpha_{j}}}{\alpha_{j}}}\right) \\
& +\rho \times \sin \left(i_{k}^{+}\right) \times \sin \left(i_{k_{2}}-\sqrt[\alpha_{j}]{\sum_{k_{j}=1}^{\alpha_{j}} \frac{\left(\mu_{k_{j}} \times i_{k_{j}}\right)^{\alpha_{j}}}{\alpha_{j}}}\right)+0+0 \\
& =\sum_{j=1}^{n} w_{j} \times\left(\rho \times\left(\sin \left(\sqrt[a]{\sum_{k_{j}=1}^{\frac{\alpha_{j}}{\left(\mu_{k_{j}} \times i_{k_{j}}\right)^{\alpha_{j}}}}}\right)-\sin \left(i_{k}^{+}\right)\right) \times \sin \left(\sqrt[a j]{\alpha_{k_{j}=1}^{\frac{\alpha_{j}}{\left(\mu_{k_{j}} \times i_{k_{j}}\right)^{\alpha_{j}}}}}-i_{k}^{+}\right)\right. \\
& \left.+\varphi \times\left(\sin \left(\sqrt[a_{j}]{\sum_{k_{j}=1}^{\alpha_{j}} \frac{\left(\eta_{k_{j}} \times i_{k_{j}}\right)^{\alpha_{j}}}{\alpha_{j}}}\right)\right)^{2}+\delta \times\left(\sin \left(\sqrt[a j]{\sum_{k_{j}=1}^{\alpha_{j}} \frac{\left(v_{k_{j}} \times i_{k_{j}}\right)^{\alpha_{j}}}{\alpha_{j}}}\right)\right)^{2}\right)
\end{aligned}
$$$$
C E_{1}^{*}\left(H_{s}, H_{s}^{-}\right)=C E_{1}\left(H_{s}, H_{s}^{-}\right)+C E_{1}\left(H_{s}^{-}, H_{s}\right)
$$$$
=\sum_{j=1}^{n} w_{j} \times\left(\rho \times \sin \left(\sqrt[a j]{\sum_{k_{j}=1}^{\alpha_{j}} \frac{\left(\mu_{k_{j}} \times i_{k_{j}}\right)^{\alpha_{j}}}{\alpha_{j}}}\right) \times \sin \left(\sqrt[a j]{\sum_{k_{j}=1}^{\alpha_{j} \frac{\left(\mu_{k_{j}} \times i_{k_{j}}\right)^{\alpha_{j}}}{\alpha_{j}}}-i_{k}^{-}}\right)+\varphi \times \sin \left(\sqrt[\alpha_{j}]{\sum_{k_{j}=1}^{\alpha_{j}} \frac{\left(\eta_{k_{j}} \times i_{k_{j}}\right)^{\alpha_{j}}}{\alpha_{j}}}\right)\right.
$$ 


$$
\begin{aligned}
& \times \sin \left(\sqrt[\alpha_{j}]{\sum_{k_{j}=1}^{\alpha_{j}} \frac{\left(\eta_{k_{j}} \times i_{k_{j}}\right)^{\alpha_{j}}}{\alpha_{j}}}\right)+\delta \times \sin \left(\sqrt[\alpha_{j}]{\sum_{k_{j}=1}^{\alpha_{j}} \frac{\left(v_{k_{j}} \times i_{k_{j}}\right)^{\alpha_{j}}}{\alpha_{j}}}\right) \times \sin \left(\sqrt[\alpha_{j}]{\sum_{k_{j}=1}^{\alpha_{j}} \frac{\left(v_{k_{j}} \times i_{k_{j}}\right)^{\alpha_{j}}}{\alpha_{j}}}\right) \\
& +\rho \times \sin \left(i_{k}^{-}\right) \times \sin \left(i_{k_{2}}-\sqrt[\alpha_{j}]{\sum_{k_{j}=1}^{\alpha_{j}} \frac{\left(\mu_{k_{j}} \times i_{k_{j}}\right)^{\alpha_{j}}}{\alpha_{j}}}\right)+0+0 \\
& =\sum_{j=1}^{n} w_{j} \times\left(\rho \times\left(\sin \left(\sqrt[\alpha_{j}]{\sum_{k_{j}=1}^{\alpha_{j}} \frac{\left(\mu_{k_{j}} \times i_{k_{j}}\right)^{\alpha_{j}}}{\alpha_{j}}}\right)-\sin \left(i_{k}^{+}\right)\right) \times \sin \left(\sqrt[a j]{\sum_{k_{j}=1}^{\alpha_{j}} \frac{\left(\mu_{k_{j}} \times i_{k_{j}}\right)^{\alpha_{j}}}{\alpha_{j}}}-i_{k}^{-}\right)\right. \\
& \left.+\varphi \times\left(\sin \left(\sqrt[a j]{\sum_{k_{j}=1}^{\alpha_{j}} \frac{\left(\eta_{k_{j}} \times i_{k_{j}}\right)^{\alpha_{j}}}{\alpha_{j}}}\right)\right)^{2}+\delta \times\left(\sin \left(\sqrt[a j]{\sum_{k_{j}=1}^{\alpha_{j}} \frac{\left(v_{k_{j}} \times i_{k_{j}}\right)^{\alpha_{j}}}{\alpha_{j}}}\right)\right)^{2}\right) .
\end{aligned}
$$

In a similar way, we can also obtain that

$$
\begin{aligned}
& C E_{2}^{*}\left(H_{s}, H_{s}^{+}\right)=C E_{2}\left(H_{s}, H_{s}^{+}\right)+C E_{2}\left(H_{s}^{+}, H_{s}\right) \\
& =\sum_{j=1}^{n} w_{j} \times\left(\rho \times\left(\tan \left(\sqrt[\alpha_{j}]{\sum_{k_{j}=1}^{\alpha_{j}} \frac{\left(\mu_{k_{j}} \times i_{k_{j}}\right)^{\alpha_{j}}}{\alpha_{j}}}\right)-\tan \left(i_{k}^{+}\right)\right) \times \tan \left(\sqrt[a j]{\sum_{k_{j}=1}^{\alpha_{j}} \frac{\left(\mu_{k_{j}} \times i_{k_{j}}\right)^{\alpha_{j}}}{\alpha_{j}}}-i_{k}^{+}\right)\right. \\
& \left.+\varphi \times\left(\tan \left(\sqrt[a j]{\sum_{k_{j}=1}^{\alpha_{j}} \frac{\left(\eta_{k_{j}} \times i_{k_{j}}\right)^{\alpha_{j}}}{\alpha_{j}}}\right)\right)^{2}+\delta \times\left(\tan \left(\sqrt[a j]{\sum_{k_{j}=1}^{\alpha_{j}} \frac{\left(v_{k_{j}} \times i_{k_{j}}\right)^{\alpha_{j}}}{\alpha_{j}}}\right)\right)^{2}\right),
\end{aligned}
$$

$C E_{2}^{*}\left(H_{s}, H_{s}^{-}\right)=C E_{2}\left(H_{s}, H_{s}^{-}\right)+C E_{2}\left(H_{s}^{-}, H_{s}\right)$

$$
\begin{aligned}
& =\sum_{j=1}^{n} w_{j} \times\left(\rho \times\left(\tan \left(\sqrt[a_{j}]{\sum_{k_{j}=1}^{\alpha_{j}} \frac{\left(\mu_{k_{j}} \times i_{k_{j}}\right)^{\alpha_{j}}}{\alpha_{j}}}\right)-\tan \left(i_{k}^{+}\right)\right) \times \tan \left(\sqrt[a_{j}]{\sum_{k_{j}=1}^{\alpha_{j}} \frac{\left(\mu_{k_{j}} \times i_{k_{j}}\right)^{\alpha_{j}}}{\alpha_{j}}}-i_{k}^{+}\right)\right. \\
& \left.+\varphi \times\left(\tan \left(\sqrt[a j]{\sum_{k_{j}=1}^{\alpha_{j}\left(\eta_{k_{j}} \times i_{k_{j}}\right)^{\alpha_{j}}}} \alpha_{j}\right)\right)^{2}+\delta \times\left(\tan \left(\sqrt[\alpha_{j}]{\sum_{k_{j}=1}^{\alpha_{j}} \frac{\left(v_{k_{j}} \times i_{k_{j}}\right)^{\alpha_{j}}}{\alpha_{j}}}\right)\right)^{2}\right),
\end{aligned}
$$

$C E_{3}^{*}\left(H_{s}, H_{s}^{+}\right)=C E_{3}\left(H_{s}, H_{s}^{+}\right)+C E_{3}\left(H_{s}^{+}, H_{s}\right)$ 


$$
\begin{aligned}
& =\sum_{j=1}^{n} w_{j} \times\left(\rho \times\left(\sqrt{\left.\sum_{k_{j}=1}^{\alpha_{j}} \frac{\left(\mu_{k_{j}} \times i_{k_{j}}\right)^{\alpha_{j}}}{\alpha_{j}}+1\right) \times \ln \frac{2 \times\left(\sqrt[\alpha j]{\sum_{k_{j}=1}^{\alpha_{j}}\left(\mu_{k_{j}} \times i_{k_{j}}\right)^{\alpha_{j}} / \alpha_{j}}+1\right)}{2+\sqrt[\alpha j]{\sum_{k_{j}=1}^{\alpha_{j}}\left(\mu_{k_{j}} \times i_{k_{j}}\right)^{\alpha_{j}} / \alpha_{j}}+i_{k}^{+}}}\right.\right. \\
& \left.+\left(i_{k}^{+}+1\right) \ln \frac{2\left(i_{k}^{+}+1\right)}{2+i_{k}^{+}+\sqrt[\alpha j]{\sum_{k_{j}=1}^{\alpha_{j}}\left(\mu_{k_{j}} \times i_{k_{j}}\right)^{\alpha_{j}} / \alpha_{j}}}\right) \\
& \cdot \varphi \times\left(\left(\sqrt[\alpha j]{\sum_{k_{j}=1}^{\alpha_{j}}\left(\eta_{k_{j}} \times i_{k_{j}}\right)^{\alpha_{j}} / \alpha_{j}}+1\right) \times \ln \frac{2 \times\left(\sqrt[\alpha j]{\sum_{k_{j}=1}^{\alpha_{j}}\left(\eta_{k_{j}} \times i_{k_{j}}\right)^{\alpha_{j}} / \alpha_{j}}+1\right)}{2+\sqrt[\alpha_{j}]{\sum_{k_{j}=1}^{\alpha_{j}\left(\eta_{k_{j}} \times i_{k_{j}}\right)^{\alpha_{j}} / \alpha_{j}}}}+\ln \frac{2}{2+\sqrt[\alpha_{j}\left(\eta_{k_{j}} \times i_{k_{j}}\right)^{\alpha_{j}} / \alpha_{j}]{\alpha_{j}}}\right) \\
& \cdot \delta \times\left(\left(\sqrt[\alpha j]{\sum_{k_{j}=1}^{\alpha_{j}}\left(v_{k_{j}} \times i_{k_{j}}\right)^{\alpha_{j}} / \alpha_{j}}+1\right) \times \ln \frac{2 \times\left(\sqrt[\alpha j]{\sum_{k_{j}=1}^{\alpha_{j}}\left(v_{k_{j}} \times i_{k_{j}}\right)^{\alpha_{j}} / \alpha_{j}}+1\right)}{2+\sqrt[\alpha j]{\sum_{k_{j}=1}^{\alpha_{j}\left(v_{k_{j}} \times i_{k_{j}}\right)^{\alpha_{j}} / \alpha_{j}}}}\right. \\
& \left.\left.+\ln \frac{2}{2+\sqrt[\alpha j]{\sum_{k_{j}=1}^{\alpha_{j}}\left(v_{k_{j}} \times i_{k_{j}}\right)^{\alpha_{j}} / \alpha_{j}}}\right)\right) \\
& C E_{3}^{*}\left(H_{s}, H_{s}^{-}\right)=C E_{3}\left(H_{s}, H_{s}^{-}\right)+C E_{3}\left(H_{s}^{-}, H_{s}\right) \\
& =\sum_{j=1}^{n} w_{j} \times\left(\rho \times\left(\sqrt{\sum_{k_{j}=1}^{\alpha_{j}} \frac{\left(\mu_{k_{j}} \times i_{k_{j}}\right)^{\alpha_{j}}}{\alpha_{j}}}+1\right) \times \ln \frac{2 \times\left(\sqrt[\alpha j]{\sum_{k_{j}=1}^{\alpha_{j}}\left(\mu_{k_{j}} \times i_{k_{j}}\right)^{\alpha_{j}} / \alpha_{j}}+1\right)}{2+\sqrt[\alpha j]{\sum_{k_{j}=1}^{\alpha_{j}}\left(\mu_{k_{j}} \times i_{k_{j}}\right)^{\alpha_{j}} / \alpha_{j}}}\right. \\
& \left.+\ln \frac{2}{2+\sqrt[\alpha j]{\sum_{k_{j}=1}^{\alpha_{j}}\left(\mu_{k_{j}} \times i_{k_{j}}\right)^{\alpha_{j}} / \alpha_{j}}}\right) \\
& \cdot \varphi \times\left(\sqrt{\sum_{k_{j}=1}^{\alpha_{j}} \frac{\left(\eta_{k_{j}} \times i_{k_{j}}\right)^{\alpha_{j}}}{\alpha_{j}}}+1\right) \times \ln \frac{2 \times\left(\sqrt[\alpha j]{\sum_{k_{j}=1}^{\alpha_{j}}\left(\eta_{k_{j}} \times i_{k_{j}}\right)^{\alpha_{j}} / \alpha_{j}}+1\right)}{2+\sqrt[\alpha j]{\sum_{k_{j}=1}^{\alpha_{j}}\left(\eta_{k_{j}} \times i_{k_{j}}\right)^{\alpha_{j}} / \alpha_{j}}} \\
& \left.+\ln \frac{2}{2+\sqrt[\alpha j]{\sum_{k_{j}=1}^{\alpha_{j}}\left(\eta_{k_{j}} \times i_{k_{j}}\right)^{\alpha_{j}} / \alpha_{j}}}\right) \\
& \cdot \delta \times\left(\sqrt{\sum_{k_{j}=1}^{\alpha_{j}} \frac{\left(v_{k_{j}} \times i_{k_{j}}\right)^{\alpha_{j}}}{\alpha_{j}}}+1\right) \times \ln \frac{2 \times\left(\sqrt[\alpha j]{\sum_{k_{j}=1}^{\alpha_{j}}\left(v_{k_{j}} \times i_{k_{j}}\right)^{\alpha_{j}} / \alpha_{j}}+1\right)}{2+\sqrt[\alpha j]{\sum_{k_{j}=1}^{\alpha_{j}}\left(v_{k_{j}} \times i_{k_{j}}\right)^{\alpha_{j}} / \alpha_{j}+i_{k}^{-}}} \\
& \left.\left.+\left(i_{k}^{-}+1\right) \ln \frac{2\left(i_{k}^{-}+1\right)}{2+i_{k}^{-}+\sqrt[\alpha j]{\sum_{k_{j}=1}^{\alpha_{j}}\left(v_{k_{j}} \times i_{k_{j}}\right)^{\alpha_{j}} / \alpha_{j}}}\right)\right)
\end{aligned}
$$




$$
\begin{aligned}
& C E_{4}^{*}\left(H_{s}, H_{s}^{+}\right)=C E_{4}\left(H_{s}, H_{s}^{+}\right)+C E_{4}\left(H_{s}^{+}, H_{s}\right) \\
& =\sum_{j=1}^{n} w_{j} \times\left(\rho \times\left(\sqrt[\alpha_{j}]{\sum_{k_{j}=1}^{\alpha_{j}} \frac{\left(\mu_{k_{j}} \times i_{k_{j}}\right)^{\alpha_{j}}}{\alpha_{j}}}-i_{k}^{+}\right) \times\left(\exp \left(\sqrt[\alpha_{j}]{\sum_{k_{j}=1}^{\alpha_{j}} \frac{\left(\mu_{k_{j}} \times i_{k_{j}}\right)^{\alpha_{j}}}{\alpha_{j}}}-1\right)-\exp \left(i_{k}^{+}-1\right)\right)\right)
\end{aligned}
$$

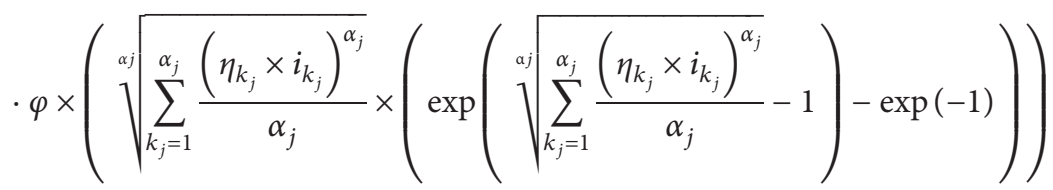

$$
\begin{aligned}
& \cdot \delta \times\left(\sqrt[\alpha_{j}]{\sum_{k_{j}=1}^{\alpha_{j}} \frac{\left(v_{k_{j}} \times i_{k_{j}}\right)^{\alpha_{j}}}{\alpha_{j}}} \times\left(\exp \left(\sqrt[\alpha_{j}]{\sum_{k_{j}=1}^{\alpha_{j}} \frac{\left(v_{k_{j}} \times i_{k_{j}}\right)^{\alpha_{j}}}{\alpha_{j}}}-1\right)-\exp (-1)\right)\right), \\
& C E_{4}^{*}\left(H_{s}, H_{s}^{-}\right)=C E_{4}\left(H_{s}, H_{s}^{-}\right)+C E_{4}\left(H_{s}^{-}, H_{s}\right) \\
& =\sum_{j=1}^{n} w_{j} \times\left(\rho \times\left(\sqrt[\alpha_{j}]{\sum_{k_{j}=1}^{\alpha_{j}} \frac{\left(\mu_{k_{j}} \times i_{k_{j}}\right)^{\alpha_{j}}}{\alpha_{j}}}-i_{k}^{-}\right) \times\left(\exp \left(\sqrt[\alpha_{j}]{\sum_{k_{j}=1}^{\alpha_{j}} \frac{\left(\mu_{k_{j}} \times i_{k_{j}}\right)^{\alpha_{j}}}{\alpha_{j}}}-1\right)-\exp \left(i_{k}^{+}-1\right)\right)\right)
\end{aligned}
$$

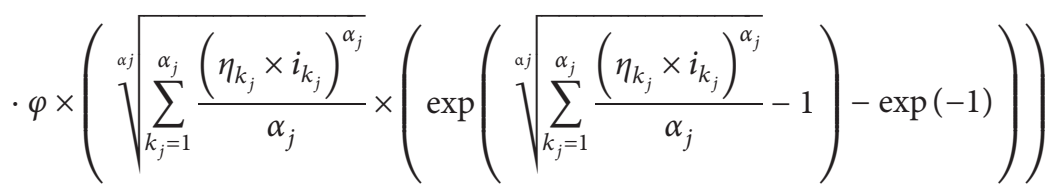

$$
\begin{aligned}
& \cdot \delta \times\left(\sqrt[\alpha_{j}]{\sum_{k_{j}=1}^{\alpha_{j}} \frac{\left(v_{k_{j}} \times i_{k_{j}}\right)^{\alpha_{j}}}{\alpha_{j}}} \times\left(\exp \left(\sqrt[\alpha_{j}]{\sum_{k_{j}=1}^{\alpha_{j}} \frac{\left(v_{k_{j}} \times i_{k_{j}}\right)^{\alpha_{j}}}{\alpha_{j}}}-1\right)-\exp (-1)\right)\right) .
\end{aligned}
$$

\subsection{The MCDM Method of HPFLS-Based Cross-Entropy and} TOPSIS. In this section, an MCDM approach of HPFLSbased cross-entropy and TOPSIS is described in detail, and the detailed decision-making steps are as follows:

Let $A=\left\{a_{1}, a_{2}, \ldots, a_{m}\right\}$ be the collection of $m$ alternatives, $E=\left\{e_{1}, e_{2}, \ldots, e_{k}\right\}$ be the collection of $k$ decisionmaker, $C=\left\{c_{1}, c_{2}, \ldots, c_{n}\right\}$ be a set of $n$ criteria, and $w=$ $\left\{w_{1}, w_{2}, \ldots, w_{n}\right\}$ be the weight collection of criteria. Next, according to the criteria, the evaluation score for alternatives $A_{i}$ on criterion $C_{j}$, which is provided by decision makers (DMs), can be expressed as decision-making matrix $M=$ $\left(\widetilde{\beta}_{i j}\right)_{m \times n}$ with HPFLSs.

The detailed decision-making steps are as follows.

Step 1. Normalize the evaluation value of the decision matrix

All criteria in the decision-making matrix must be distinguished as benefit-type or cost-type criteria. In order to normalize the criteria values, normally, the evaluation values of the benefit criteria do not need to be changed, and the evaluation values of the cost criteria need to be replaced with their complementary sets.
The following formula is used to normalize the decisionmaking matrix:

$$
\tilde{\tilde{\beta}}_{i j}= \begin{cases}\tilde{\beta}_{i j}, & C_{j} \in B_{S}, \\ \tilde{\beta}_{i j}^{c}, & C_{j} \in C_{S},\end{cases}
$$

where $B_{S}$ is the set of benefit criteria, $C_{S}$ is the set of cost criteria, and $\widetilde{\beta}_{i j}^{c}$ is the complementary set of $\widetilde{\beta}_{i j}$. The normalized decision-making matrix is denoted by $\widehat{M}=\left(\tilde{\tilde{\beta}}_{i j}\right)_{m \times n}$.

Step 2. Calculate the vector of criteria weight

Due to the unknown weight $w_{j}$ of criteria $c_{j}(j=1,2$, $\ldots, n)$, the value of the criteria weight needs to be firstly determined. In accordance with information theory, if the criterion has similar or the same value for all schemes, then the criterion is assigned with smaller weight because these criteria are less helpful to distinguish the advantage and disadvantage of alternatives. Then, according to formula (19), the weight value $w_{j}$ of each criterion $C_{j}$ can be obtained.

Step 3. Calculate the score of each alternative

According to the formulas in Section 4.2, the weighted cross-entropy values from each alternative to the positive 
ideal solution $\quad H_{s}^{+}=\overbrace{\left\{\left\langle\left(s_{\max }\right),(1,0,0)\right\rangle, \ldots,\left\langle\left(s_{\max }\right)\right.\right.}^{n}$, $(1,0,0)\rangle\}$ and the negative ideal solution $H_{s}^{-}=\overbrace{\left\{\left\langle\left(s_{\min }\right)\right.\right.}$, $\left.(0,0,1)\rangle, \ldots,\left\langle\left(s_{\min }\right),(0,0,1)\right\rangle\right\}$ under hesitant picture fuzzy linguistic environment are gained. Based on the value of weighted cross-entropy, the score value of each alternative can be obtained by the following formula:

$$
R_{i}=\frac{C E_{j}^{*}\left(H_{s_{i}}, H_{s}^{+}\right)}{C E_{j}^{*}\left(H_{s_{i}}, H_{s}^{+}\right)+C E_{j}^{*}\left(H_{s_{i}}, H_{s}^{-}\right)}, \quad j=1,2,3,4 .
$$

Step 4. Rank the alternative with ordered scores

The obtained score $R$ is ordered from small to large. The smaller the score is, the better the alternative is, and vice versa.

\section{Practical Application}

In this section, the middle-level managers' selection of Dongfeng Commercial Vehicle Co., Ltd., with the approach of weighted cross-entropy of HPFLSs is introduced, and the further analysis is presented.

5.1. Illustration of Practical Application. Practical application of the recruitment of middle-level management personnel in Dongfeng Commercial Vehicle Co., Ltd., is adopted as the illustration of the proposed method. Dongfeng Commercial Vehicle Co., Ltd., is the largest commercial vehicle manufacturer in China, which is mainly engaged in the production of heavy trucks, engines, gearboxes, and so forth. Due to the demands of business development, the company needed to recruit professional personnel as the company's middle-level managers with automotive engineering background and high-level automotive marketing capabilities. After several rounds of interviews, four candidates were selected for the final interview, and the evaluation of each candidate was scored from four criteria, which are negotiation and communication skills $c_{1}$, business development capability $c_{2}$, sales and marketing capability $c_{3}$, and technical background $c_{4}$. Weight for each criterion was not given in advance. Ten higher-level managers from the company are chosen as the interviewers; they are from HR, technical, sales, and financial department; all interviewers discussed and gave the evaluation value, which are collected as shown in Table 1.

Step 1: normalize the evaluation value of decisionmaking matrix

All criteria are identified as benefit type, so the decision-making matrix does not need to be normalized

Step 2: calculate the weight vector for criteria

Since the weights of the criteria are completely unknown, formula (20) is used to obtain the weight vector as $w=(0.2875,0.4938,0.1250,0.0938)$

Step 3: calculate the score of each alternative According to the formula in Section 1.3.2, the weighted cross-entropy value of HPFLSs from each alternative to PIS $H_{s}^{+}=\overbrace{\left\{\left\langle\left(s_{\max }\right),(1,0,0)\right\rangle, \ldots,\left\langle\left(s_{\max }\right),(1,0,0)\right\rangle\right\}}^{n}$ and NIS $H_{s}^{-}=\overbrace{\left\{\left\langle\left(s_{\min }\right),(0,0,1)\right\rangle, \ldots,\left\langle\left(s_{\min }\right),(0,0,1)\right\rangle\right\}}$ can be obtained. For the convenience of demonstration, the parameter is assigned with $\theta=4, n=1$, thus, the results of each alternative with four cross-entropy formulas are obtained as follows:

$$
\begin{aligned}
& R_{1}=\frac{C E_{1}^{*}\left(H_{s_{1}}, H_{s}^{+}\right)}{C E_{1}^{*}\left(H_{s_{1}}, H_{s}^{+}\right)+C E_{1}^{*}\left(H_{s_{1}}, H_{s}^{-}\right)}=\frac{0.1712}{0.1712+0.8645}=0.1653, \\
& R_{1}=\frac{C E_{2}^{*}\left(H_{s_{1}}, H_{s}^{+}\right)}{C E_{2}^{*}\left(H_{s_{1}}, H_{s}^{+}\right)+C E_{2}^{*}\left(H_{s_{1}}, H_{s}^{-}\right)}=\frac{0.4649}{0.4649+2.1757}=0.1761, \\
& R_{1}=\frac{C E_{3}^{*}\left(H_{s_{1}}, H_{s}^{+}\right)}{C E_{3}^{*}\left(H_{s_{1}}, H_{s}^{+}\right)+C E_{3}^{*}\left(H_{s_{1}}, H_{s}^{-}\right)}=\frac{0.0365}{0.0365+0.1941}=0.1583, \\
& R_{1}=\frac{C E_{4}^{*}\left(H_{s_{1}}, H_{s}^{+}\right)}{C E_{4}^{*}\left(H_{s_{1}}, H_{s}^{+}\right)+C E_{4}^{*}\left(H_{s_{1}}, H_{s}^{-}\right)}=\frac{0.1678}{0.1678+0.6810}=0.1977 .
\end{aligned}
$$

In similar way, the value of $R_{2}, R_{3}$, and $R_{4}$ can be obtained. The detailed results are shown in Table 2.

Step 4: rank the alternative with ordered scores

According to the obtained scores $R_{i}(i=1,2,3,4)$, the scores are ordered from high to low. The lower the score is, the better the alternative is. It is obviously to see that if crossentropy $C E_{4}^{*}$ is used, the alternative order is $a_{1}>a_{3}>a_{4}>a_{2}$; if the cross-entropy $C E_{1}^{*}, C E_{2}^{*}$, or $C E_{3}^{*}$ is used, the alternative ranking is $a_{3}>a_{1}>a_{4}>a_{2}$.

5.2. Further Analysis. In order to further analyze the influence of each alternative value $R_{i}(i=1,2,3,4)$ and its ranking with four different cross-entropy formulas under different parameter values environment, the alternative 


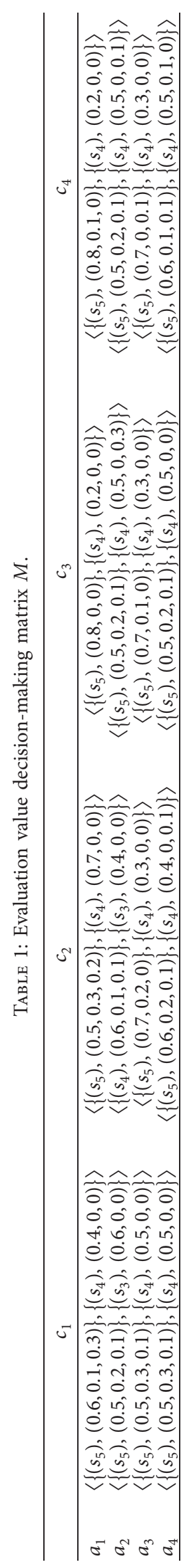


TABLE 2: Score of each alternative.

\begin{tabular}{lcccr}
\hline & $R_{1}$ & $R_{2}$ & $R_{3}$ & $R_{4}$ \\
\hline$C E_{1}^{*}$ & 0.1653 & 0.1915 & 0.1614 & 0.1884 \\
$C E_{2}^{*}$ & 0.1761 & 0.2077 & 0.1678 & 0.2014 \\
$C E_{3}^{*}$ & 0.1583 & 0.1797 & 0.1487 & 0.1775 \\
$C E_{4}^{*}$ & 0.1977 & 0.2366 & 0.2020 & 0.2297 \\
\hline
\end{tabular}

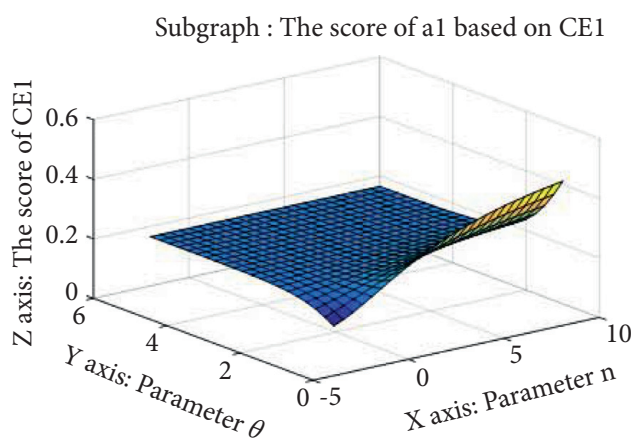

Subgraph : The score of a1 based on CE3

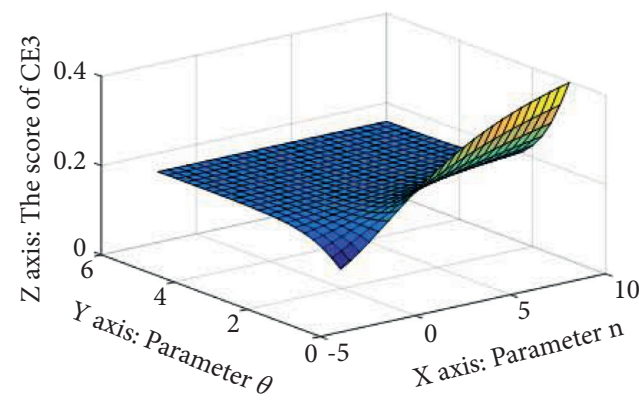

Subgraph : The score of a1 based on CE2

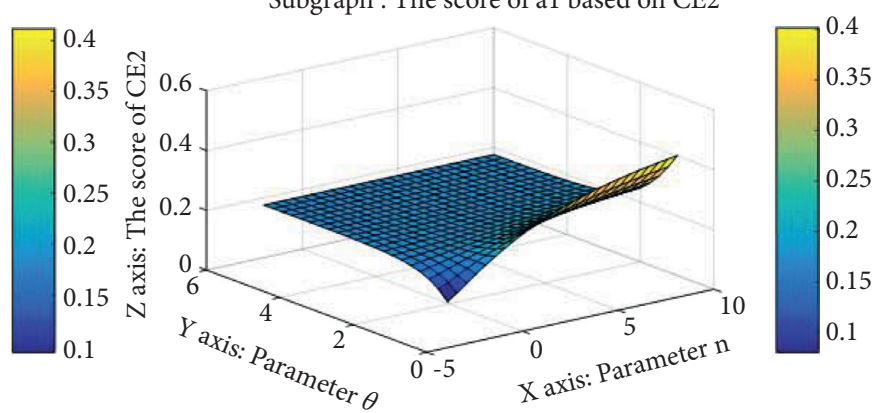

Subgraph : The score of al based on CE4

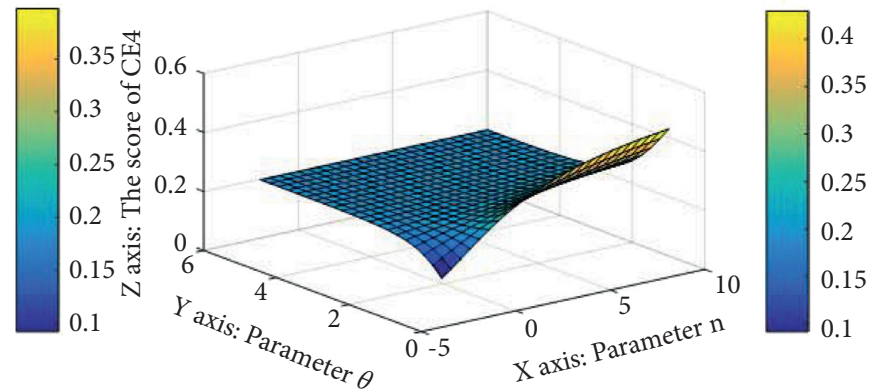

Figure 2: Score of alternative $a_{1}$ with parameter values $1 \leq \theta \leq 6,-2 \leq n \leq 10$.

Subgraph : The score of a2 based on CE1

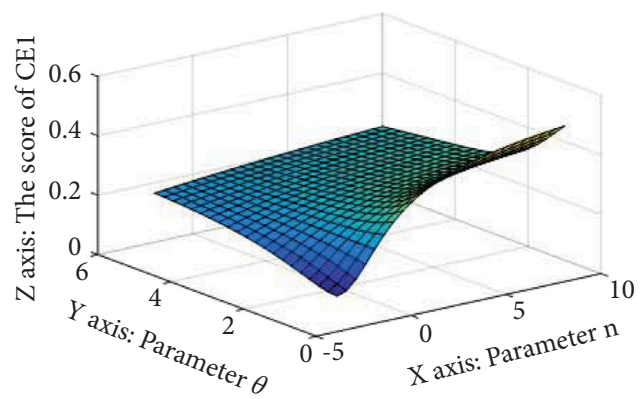

Subgraph : The score of a2 based on CE3

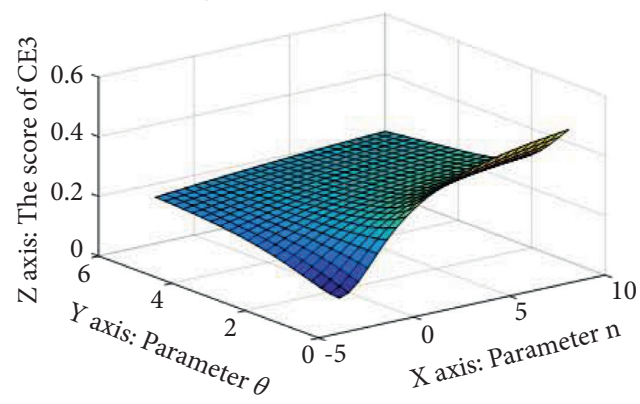

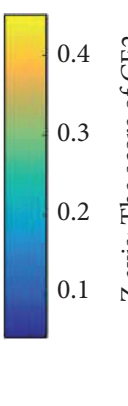

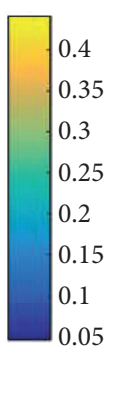

Subgraph : The score of a2 based on CE2

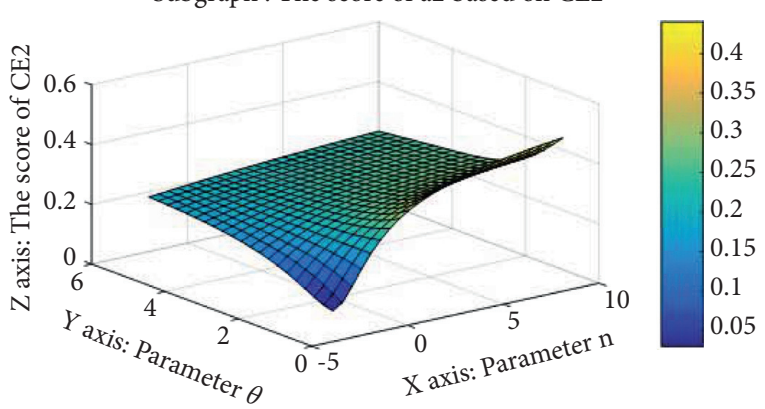

Subgraph : The score of a2 based on CE4

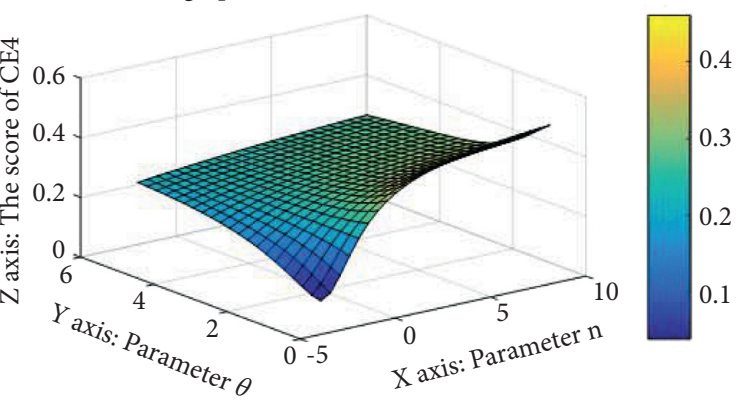

Figure 3: Score of alternative $a_{2}$ based on parameter values $1 \leq \theta \leq 6,-2 \leq n \leq 10$. 
Subgraph : The score of a 3 based on CE1

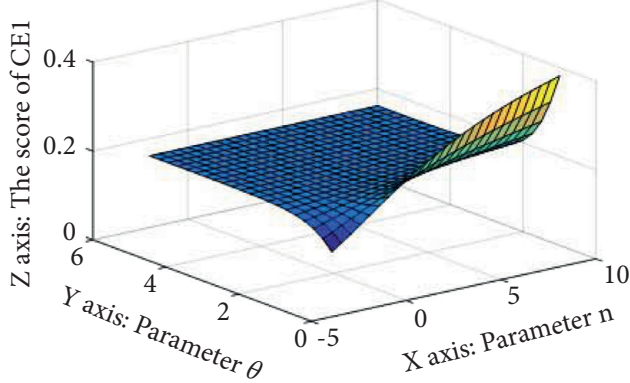

Subgraph : The score of a 3 based on CE3

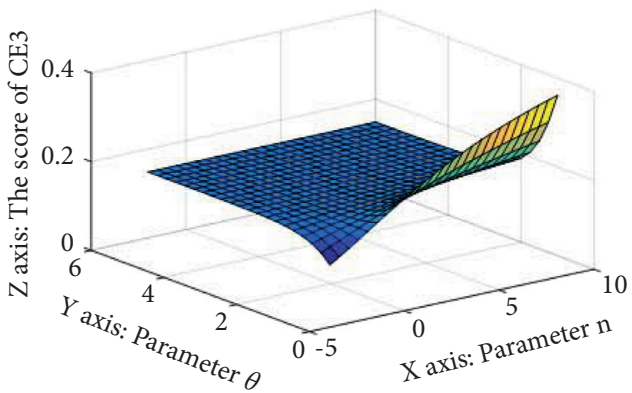

Subgraph : The score of a3 based on CE2
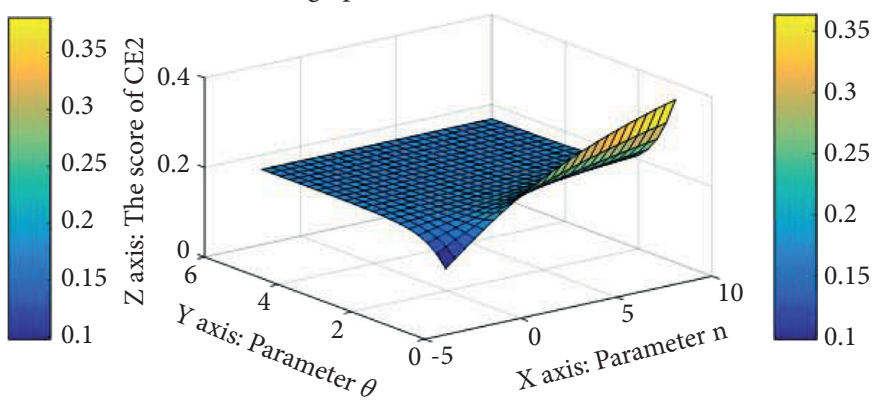

Subgraph : The score of a3 based on CE4

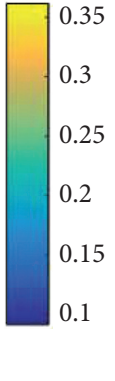

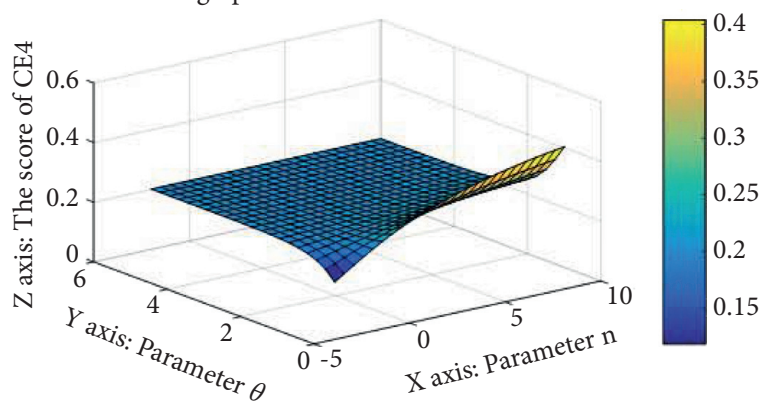

Figure 4: Score of alternative $a_{3}$ based on parameter values $1 \leq \theta \leq 6,-2 \leq n \leq 10$.

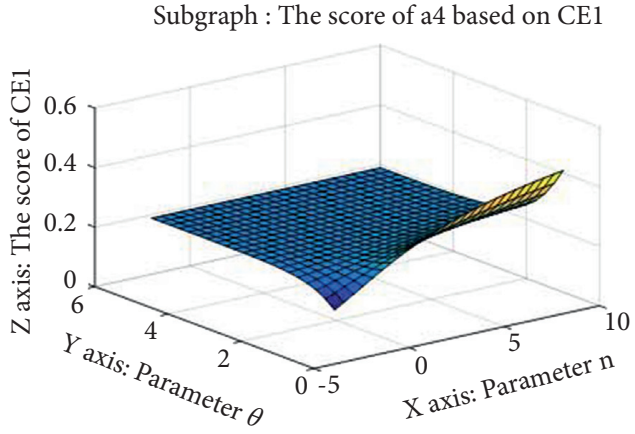

Subgraph : The score of a 4 based on CE3

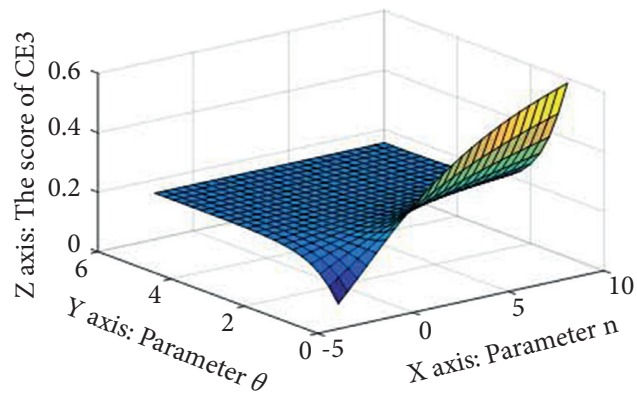

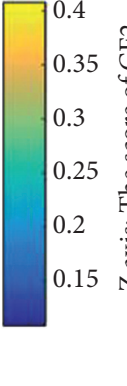

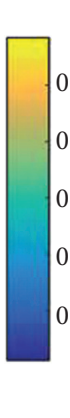

Subgraph : The score of a4 based on CE2

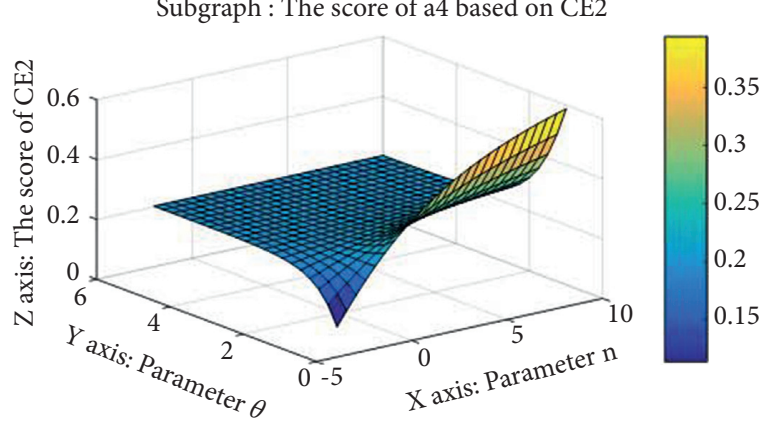

Subgraph : The score of a 4 based on CE4

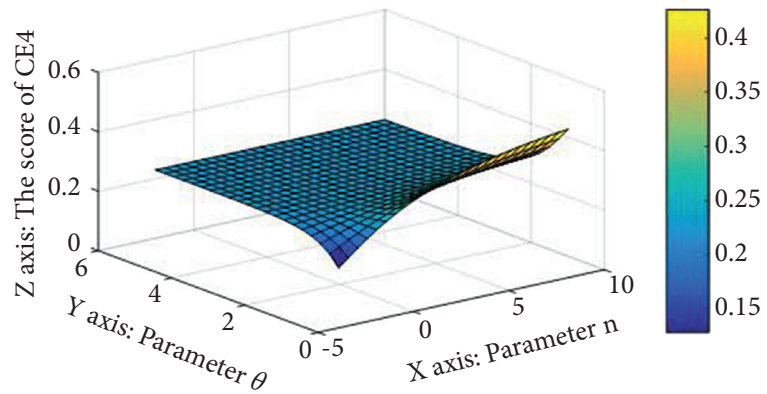

FigURE 5: Score of alternative $a_{4}$ based on parameter values $1 \leq \theta \leq 6,-2 \leq n \leq 10$.

ranking of the overall trend with different parameter combinations are conducted. The detailed alternative score is shown as follows:

According to the results of each subgraph in Figures 2-5, the over trend of alternative scores is similar for different cross-entropies with parameter values $1 \leq \theta \leq 6$ and $-2 \leq n \leq 10$. If parameter $\theta$ approaches to 1 and $n$ approaches to -2 , the score of each scheme is minimum; when parameter $\theta$ approaches to 1 and $n$ approaches to 10 , the score of each alternative is maximum. The difference is that if the parameters $\theta$ and $n$ are assigned with the same value, the increasing or decreasing value of each alternative score is different, which is mainly caused by the usage of different functions for each cross-entropy.

In order to easily understand the influence of parameters $\theta$ and $n$ on the ranking score of alternative, the fixed values 

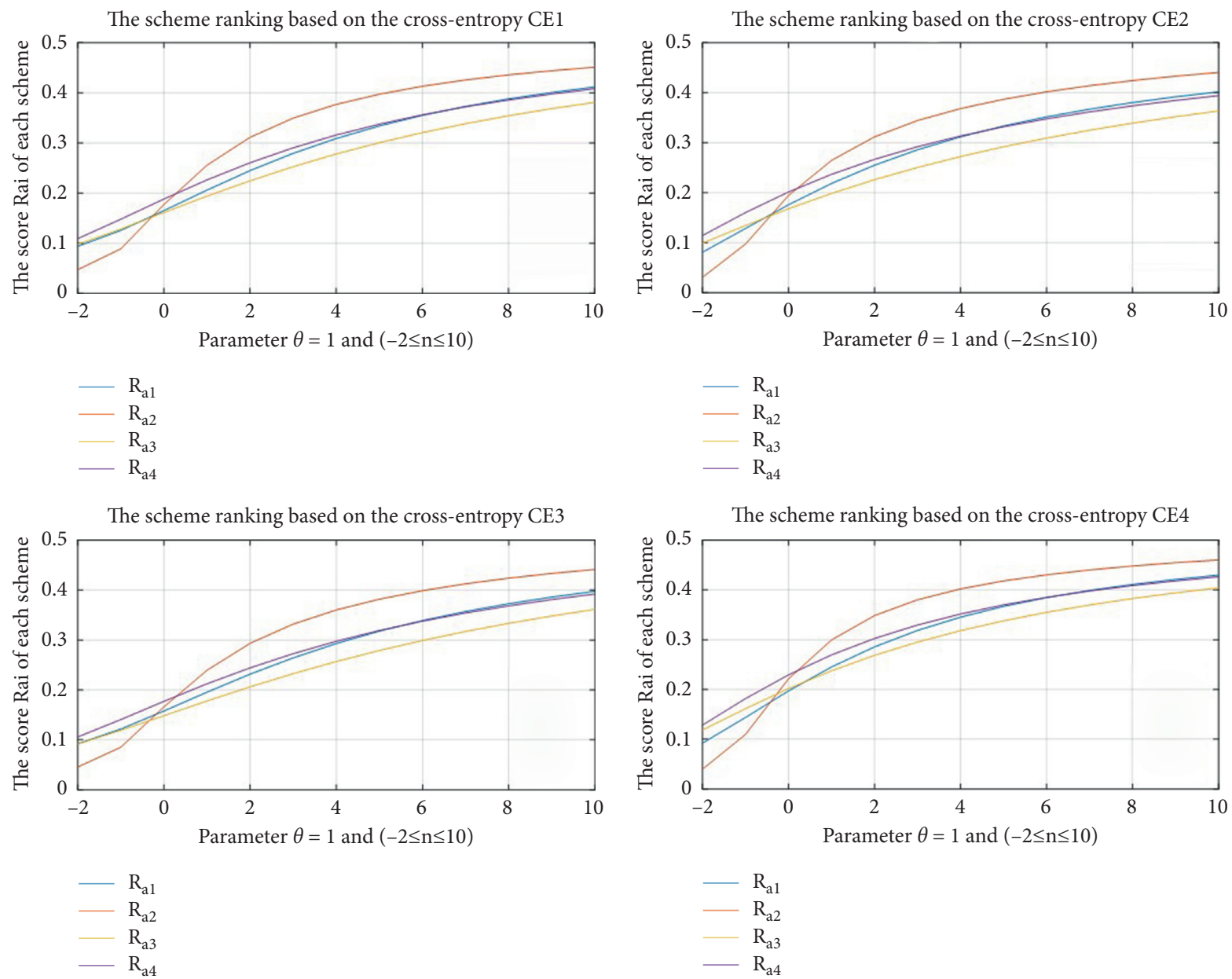

FIGURE 6: Ranking score of each alternative based on parameters $\theta=1,-2 \leq n \leq 10$.

are assigned for parameter $\theta$, that is, $\theta=1,2,5,10$, and the parameter $n$ value range is assigned with $-2 \leq n \leq 10$. The ranking score trends of each alternative with four different cross-entropy formulas are shown in Figures 6-9 as follows.

As shown in Figures 6-9, the smaller the value of parameter $\theta$ is, the value change of the parameter $n$ has a significant influence on the score and ranking results of each alternative. However, as the value of parameter $\theta$ is increasing, the value changes of parameter $n$ are less and less able to influence the scores and ranking results of each alternative.

Next, the ranking score results of each alternative influenced by the parameter value range $1 \leq \theta \leq 8$ are analyzed under the condition in which the value of parameter $n$ is fixed, that is, $n=-2,0,3,8,18$. The detailed results are shown in Figures 10-14 as follows.

From the ranking and score results presented in Figures $10-14$, it is obvious to see that if $n<0$, with the increasing of parameter value $\theta$, the score of each alternative is increasing and the increasing amplitude is different. Meanwhile, the ranking order of each alternative also changes. If $n=0$, the ranking value of each alternative will not change with the increasing value of parameter $\theta$, and the ranking order of each alternative is relatively fixed. If $n>0$, the score of each alternative is decreasing, and the final ranking results change slightly as the increasing value of parameter $\theta$.

If $n<0$ and $\theta<5$, the best alternative is $a_{4}$, the worst alternative is $a_{2}$; if $\theta>5$, the best alternative is still $a_{1}$, but the worst alternative becomes $a_{3}$ or $a_{1}$.

If $n<0$, the best alternative is $a_{4}$, and the worst alternative is $a_{3}$ or $a_{1}$.

If $a_{k}$, with the increasing of the value of parameter $\vartheta\left(a_{i}, a_{k}\right)=\left[\begin{array}{cccc}0 & -0.0691 & -0.0457 & -0.0537 \\ -0.0134 & 0 & -0.0061 & -0.0074 \\ -0.0220 & -0.0448 & 0 & -0.0210 \\ -0.0069 & -0.0400 & -0.0085 & 0\end{array}\right]$, the best alternative is $a_{2}$, but the worst alternative is $a_{3}$ or $a_{1}$.

From the above analysis of Figures 9-13, the following conclusions can be obtained:

(i) If the value of parameter $\theta$ is fixed, while the value of parameter $n$ increases, the score of the alternatives become bigger, which indicates that the criteria with highest evaluation value can bring biggest influence for the ranking order of alternatives, and vice versa.

(ii) If parameter $n<0$ and its values are fixed, with the continuous increasing of parameter $\theta$, the score of 
The scheme ranking based on the cross-entropy CE1

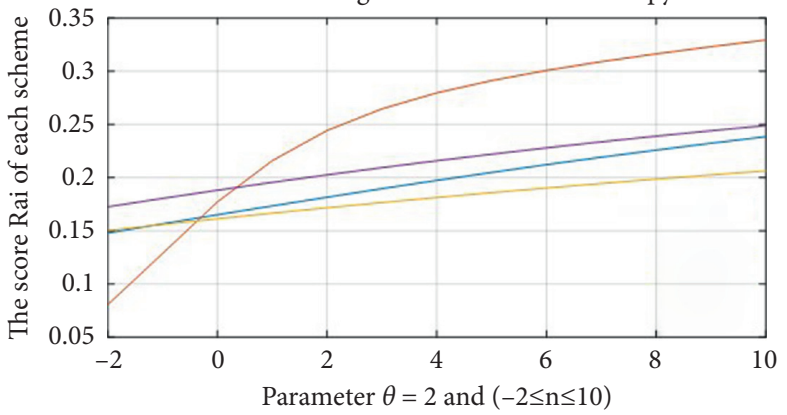

$-\mathrm{R}_{\mathrm{a} 1}$

$-\mathrm{R}_{\mathrm{a} 2}$

$-\mathrm{R}_{\mathrm{a} 3}$

$-\mathrm{R}_{\mathrm{a} 4}$

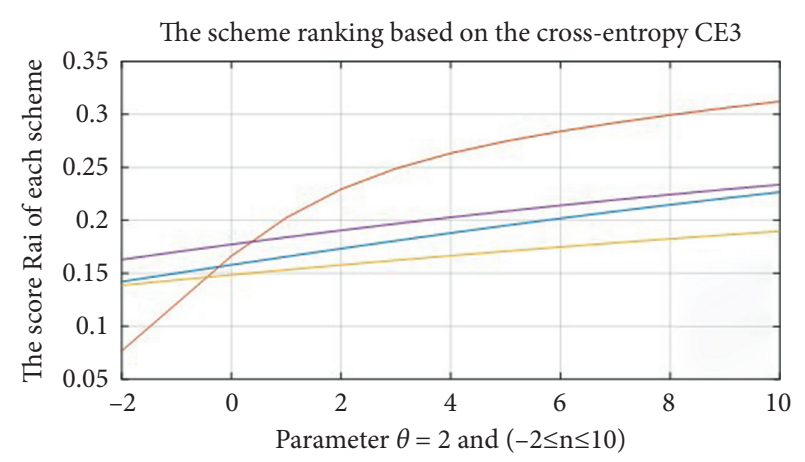

$-\mathrm{R}_{\mathrm{a} 1}$
$-\mathrm{R}_{\mathrm{a} 2}$
$\mathrm{R}_{\mathrm{a} 3}$
$-\mathrm{R}_{\mathrm{a} 4}$
The scheme ranking based on the cross-entropy CE2

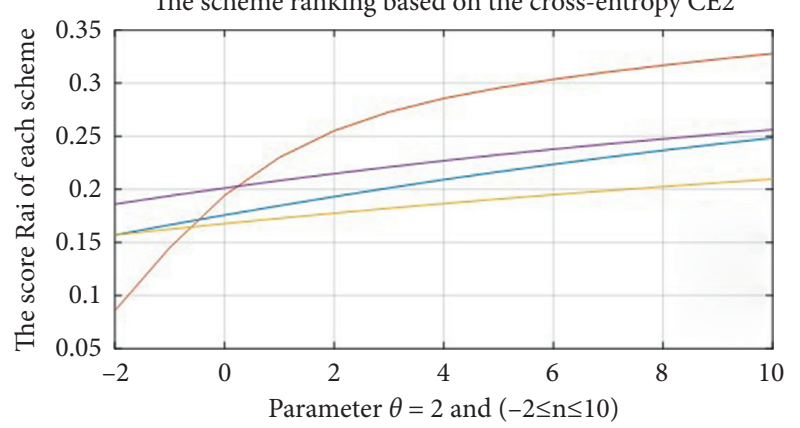

$-\mathrm{R}_{\mathrm{a} 1}$

$-\mathrm{R}_{\mathrm{a} 2}$

$-\mathrm{R}_{\mathrm{a} 3}$

$-\mathrm{R}_{\mathrm{a} 4}$

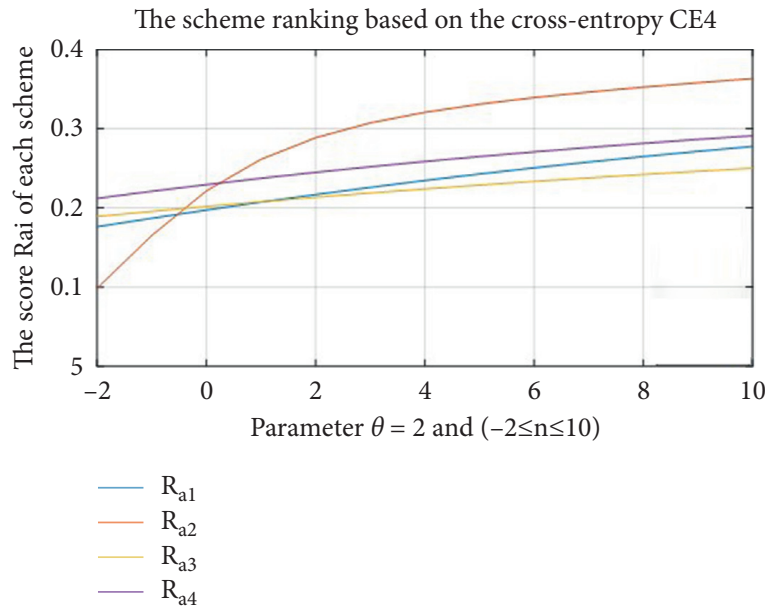

FIGURE 7: Ranking score of each alternative based on parameters $\theta=2,-2 \leq n \leq 10$.

the alternative grows bigger slightly, which indicates that the integration value of each alternative is more influenced by the criterion with high evaluation value, and vice versa.

(iii) If parameter $n>0$ and its values are fixed, with the continuous increasing of parameter $\theta$, the score of the alternative become smaller, which indicates that the integration value of each alternative is more influenced by the criteria with the low evaluation value.

From the above analysis results, considering practical applications, decision-makers pay more attention to the final ranking results affected by the criteria with high evaluation values. The parameter value $n<0$ is selected, and the value of parameter $\theta$ should be as large as possible. If decisionmakers pay more attention to the criteria with low evaluation values which affect the final ranking results, the parameter value $n>0$ is selected, and the value of parameter $\theta$ should also be as large as possible.
5.3. Comparative Analysis. In order to verify the effectiveness of the constructed approach, the comparative analysis is carried out with the representative method of PFS-based weighted cross-entropy, which is proposed by Wei [28]. For convenient comparison, the HPFLSs have been transformed to PFS. In accordance with the further analysis presented in Subsection 5.2, four pairs of representative values for parameter $\theta$ and $n$ are selected. Then, the comparative results for the different methods are shown in Table 3.

Table 3 shows that the ranking result obtained by Wei [28] has the difference with obtained by our methods. The reasons causing the difference mainly are the parameters, that is, there is no parameter in Wei's study [28]. However, there are two parameters in our proposed methods. For our methods, if different pairs of values for parameter $\theta$ and $n$ are chosen, the final ranking of alternative is slightly different. In some cases, alternative $a_{4}$ is superior to alternative $a_{2}$; in some cases, alternative $a_{2}$ is superior to alternative $a_{4}$. The reasons causing different ranking results are discussed in Section 5.2. 

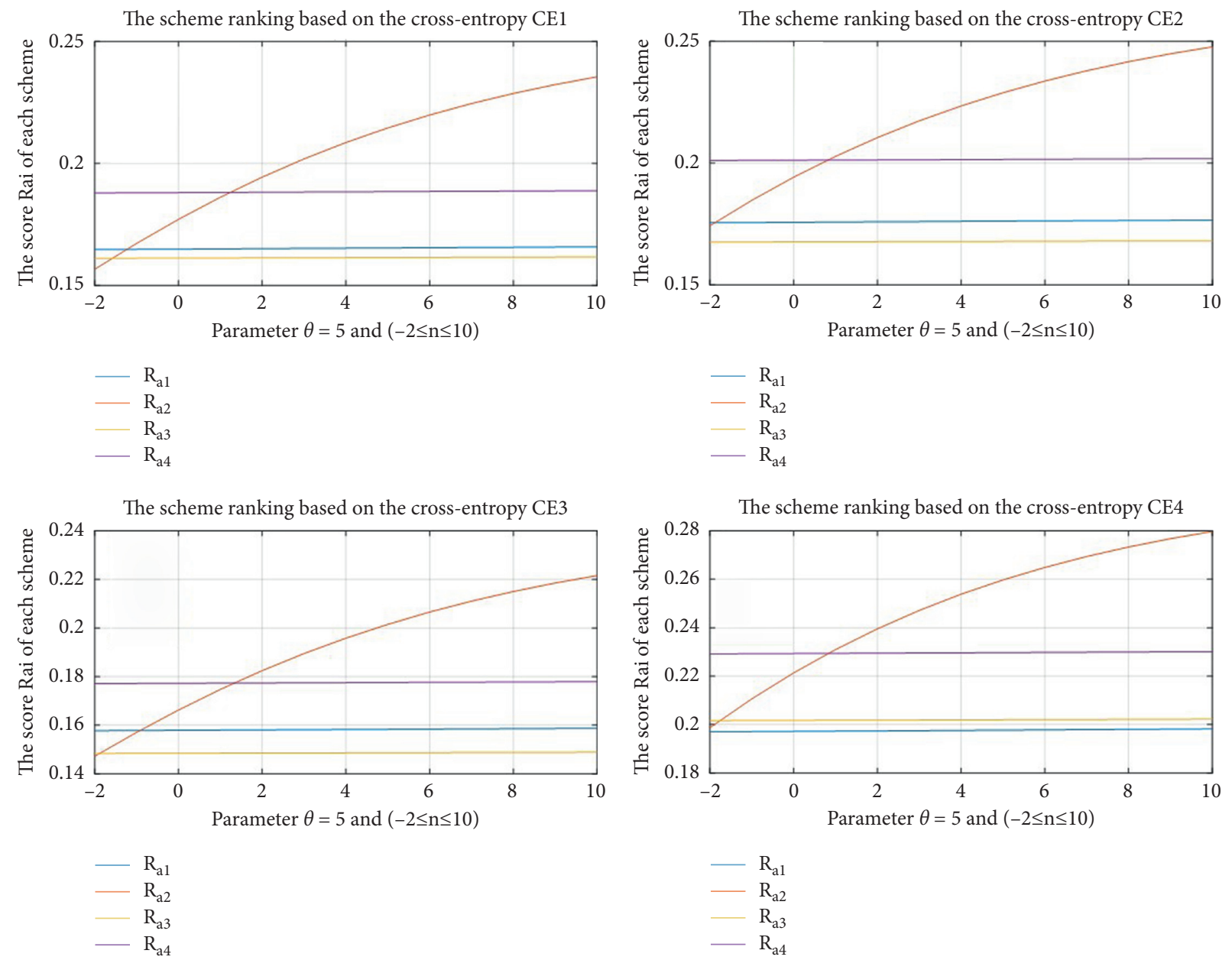

FIGURE 8: Ranking score of each alternative based on parameters $\theta=5,-2 \leq n \leq 10$.

In accordance with the comparative analysis and further discussion in Section 5.2, it can be concluded that our proposed method owns the advantages as follows:

(1) The proposed method elaborates the advantages of hesitant fuzzy set and picture fuzzy linguistic set and is better to describe the hesitant degree and risk attitude information of DM

(2) In accordance with the information theory, the criteria weights in the proposed method are obtained by the maximum-minimum distance algorithm; thus, the results are more objective and precise

(3) Under the practical decision-making environments, some DMs might pay higher attention to membership, such as membership degree of "vote for" or "vote against," and the constructed approach is more flexible and reasonable to provide the different ranking results by adjusting the value of the parameter $\theta$ and $n$; thus, the more acceptable results are provided for DMS.

\section{Conclusion and Further Direction}

Considering the actual personnel selection decision-making problem, different DMs could have different attitudes (e.g., attitude for support, neutral, oppose, and refusal) for the evaluation value of criteria or alternative and might pay high attention to the influence of job candidate ranking by the criteria with high or low evaluation value; meanwhile, the criteria weight objectively obtained by criteria evaluation value is more reasonable than subjectively given by experts; thus, an approach to elaborate the advantages of both crossentropy and HPFLSs is proposed.

The main contribution of the paper is defining the cross-entropy of HPFLN and listing its properties needed to be satisfied. Based on the definition of cross-entropy of HPFLN, several formulas of cross-entropy of HPFLN are constructed, and the relative properties are proven. In accordance with the proposed cross-entropy formula, an MCDM approach based on cross-entropy and TOPSIS under the HPFLSs environment are constructed. Finally, the real personnel selection decision-making problem from Dongfeng Commercial Vehicle Co., Ltd., is used to verify the proposed method and demonstrate effectiveness.

The limitation of our proposed method is in most reallife MCGDM environment; DMs more prefer to provide the evaluation value with simple crisp number or linguistic term; thus, how to transfer the simple crisp 

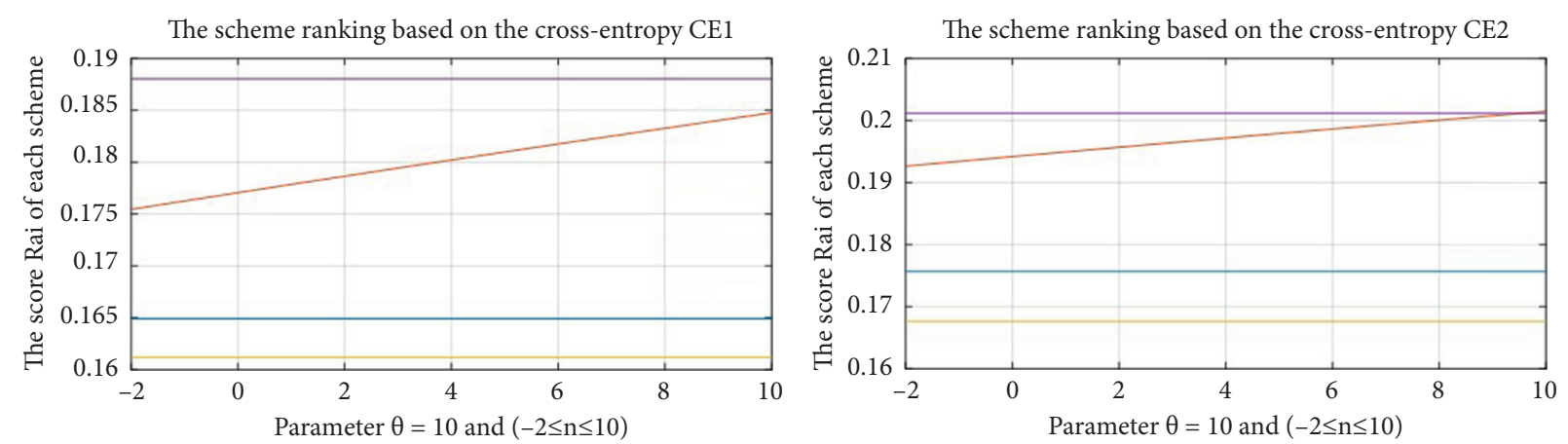

$$
\begin{array}{r}
-\mathrm{R}_{\mathrm{a} 1} \\
-\mathrm{R}_{\mathrm{a} 2} \\
-\mathrm{R}_{\mathrm{a} 3} \\
-\mathrm{R}_{\mathrm{a} 4}
\end{array}
$$

$$
\begin{array}{r}
-\mathrm{R}_{\mathrm{a} 1} \\
-\mathrm{R}_{\mathrm{a} 2} \\
-\mathrm{R}_{\mathrm{a} 3} \\
-\mathrm{R}_{\mathrm{a} 4}
\end{array}
$$
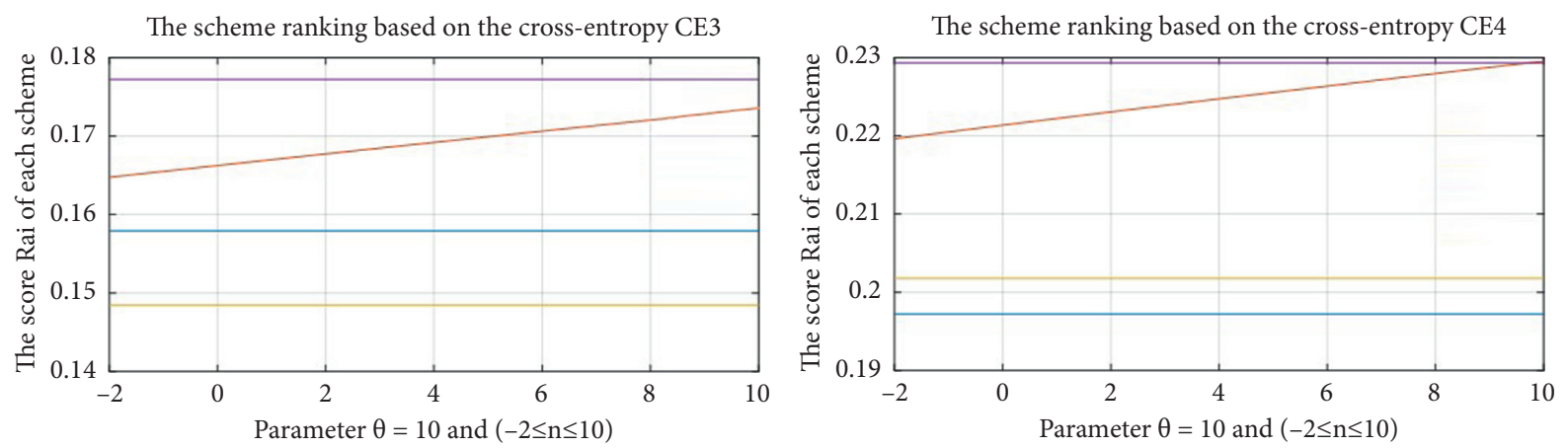

$$
\begin{array}{r}
-\mathrm{R}_{\mathrm{a} 1} \\
-\mathrm{R}_{\mathrm{a} 2} \\
-\mathrm{R}_{\mathrm{a} 3} \\
-\mathrm{R}_{\mathrm{a} 4}
\end{array}
$$$$
\begin{aligned}
- & \mathrm{R}_{\mathrm{a} 1} \\
- & \mathrm{R}_{\mathrm{a} 2} \\
- & \mathrm{R}_{\mathrm{a} 3} \\
- & \mathrm{R}_{\mathrm{a} 4}
\end{aligned}
$$

Figure 9: Ranking score of each alternative based on parameters $\theta=10,-2 \leq n \leq 10$. 

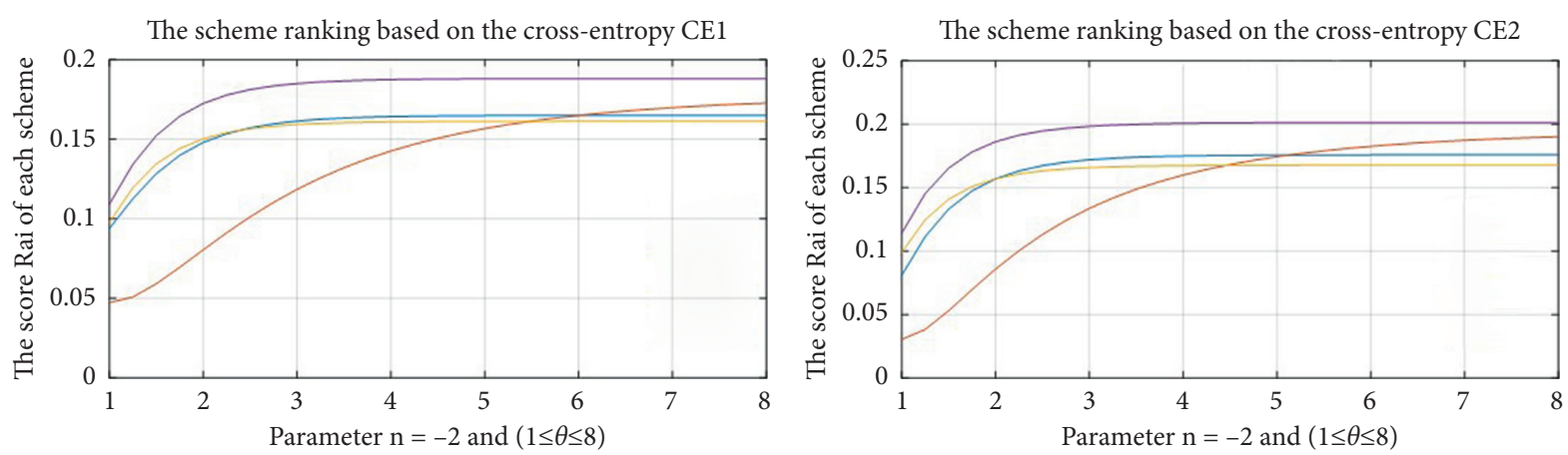

$$
\begin{array}{r}
-\mathrm{R}_{\mathrm{a} 1} \\
-\mathrm{R}_{\mathrm{a} 2} \\
-\mathrm{R}_{\mathrm{a} 3} \\
-\mathrm{R}_{\mathrm{a} 4}
\end{array}
$$

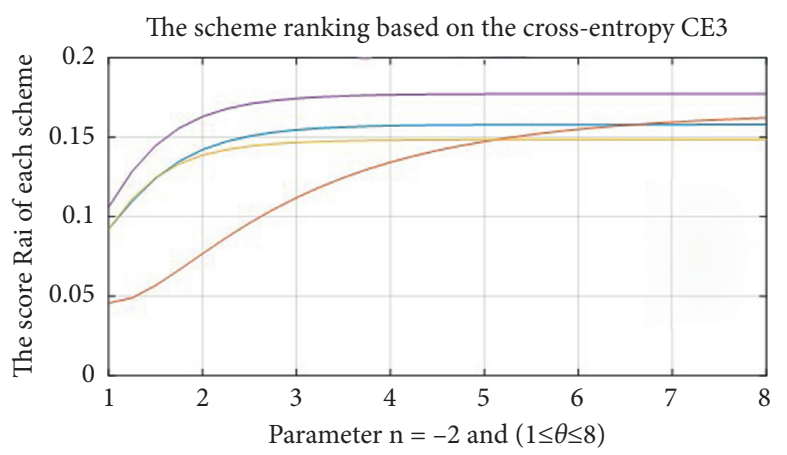

$$
\begin{array}{r}
-\mathrm{R}_{\mathrm{a} 1} \\
-\mathrm{R}_{\mathrm{a} 2} \\
-\mathrm{R}_{\mathrm{a} 3} \\
-\mathrm{R}_{\mathrm{a} 4}
\end{array}
$$

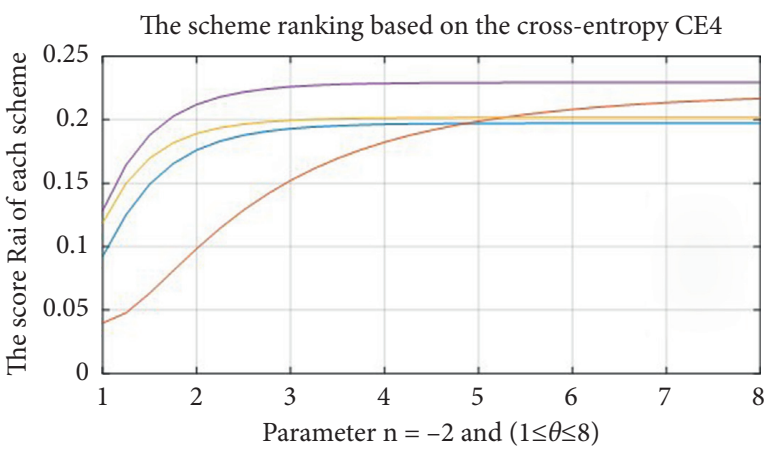

$$
\begin{array}{r}
-\mathrm{R}_{\mathrm{a} 1} \\
-\mathrm{R}_{\mathrm{a} 2} \\
-\mathrm{R}_{\mathrm{a} 3} \\
-\mathrm{R}_{\mathrm{a} 4}
\end{array}
$$$$
\text { - } \mathrm{R}_{\mathrm{a} 1}
$$$$
-\mathrm{R}_{\mathrm{a} 2}
$$$$
\text { - } \mathrm{R}_{\mathrm{a}}
$$

FIGURE 10: Ranking score of each alternative based on parameters $n=-2,1 \leq \theta \leq 8$. 

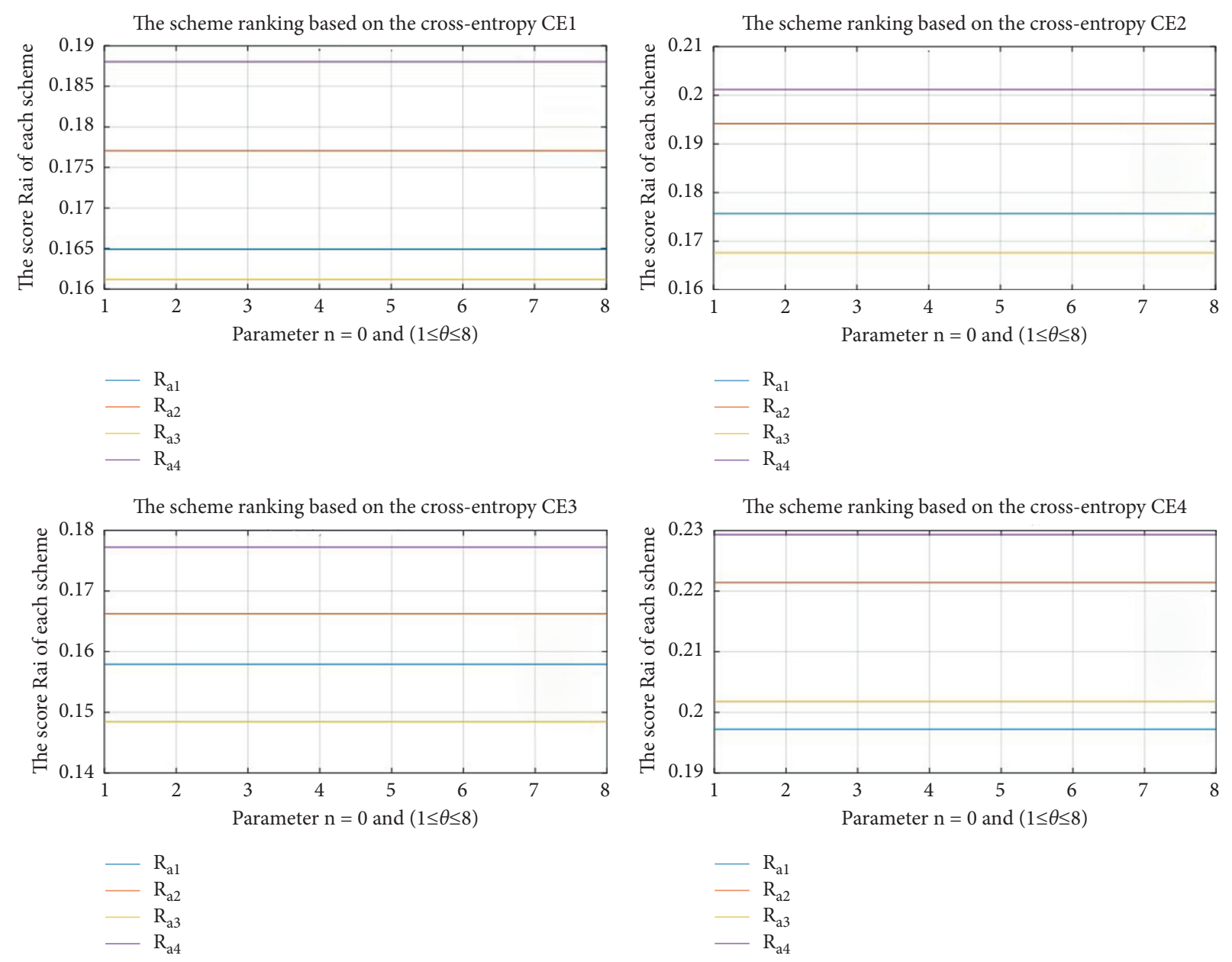

FIGURE 11: Ranking score of each alternative based on parameters $n=0,1 \leq \theta \leq 8$. 

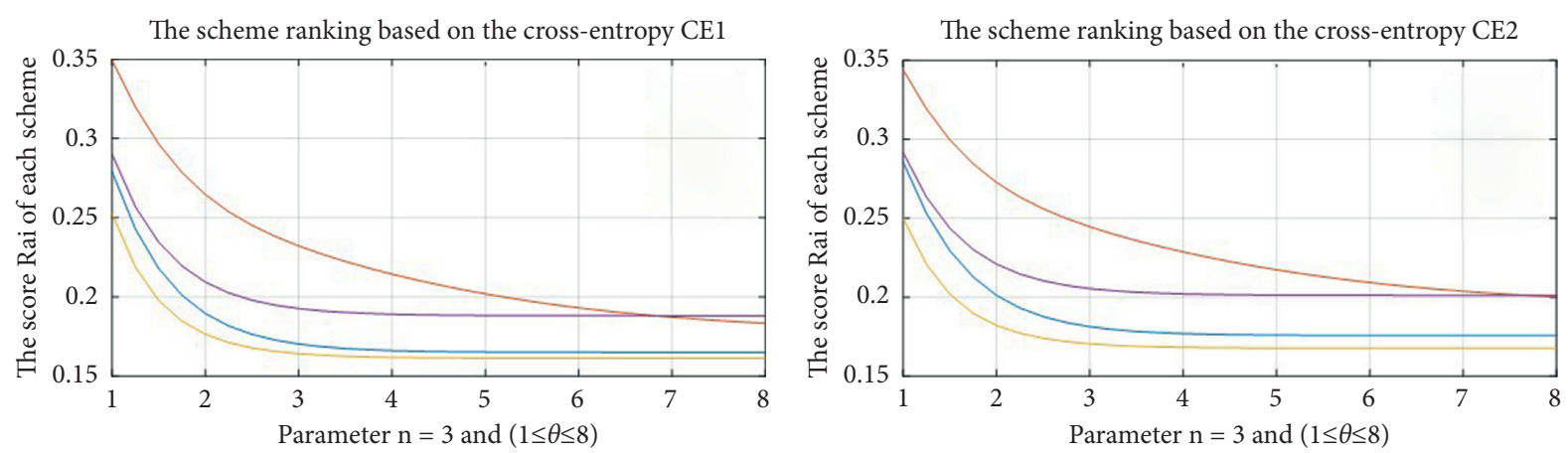

$$
\begin{aligned}
& -\mathrm{R}_{\mathrm{a} 1} \\
& -\mathrm{R}_{\mathrm{a} 2} \\
& -\mathrm{R}_{\mathrm{a} 3} \\
& -\mathrm{R}_{\mathrm{a} 4}
\end{aligned}
$$

$$
\begin{array}{r}
-\mathrm{R}_{\mathrm{a} 1} \\
-\mathrm{R}_{\mathrm{a} 2} \\
-\mathrm{R}_{\mathrm{a} 3} \\
-\mathrm{R}_{\mathrm{a} 4}
\end{array}
$$
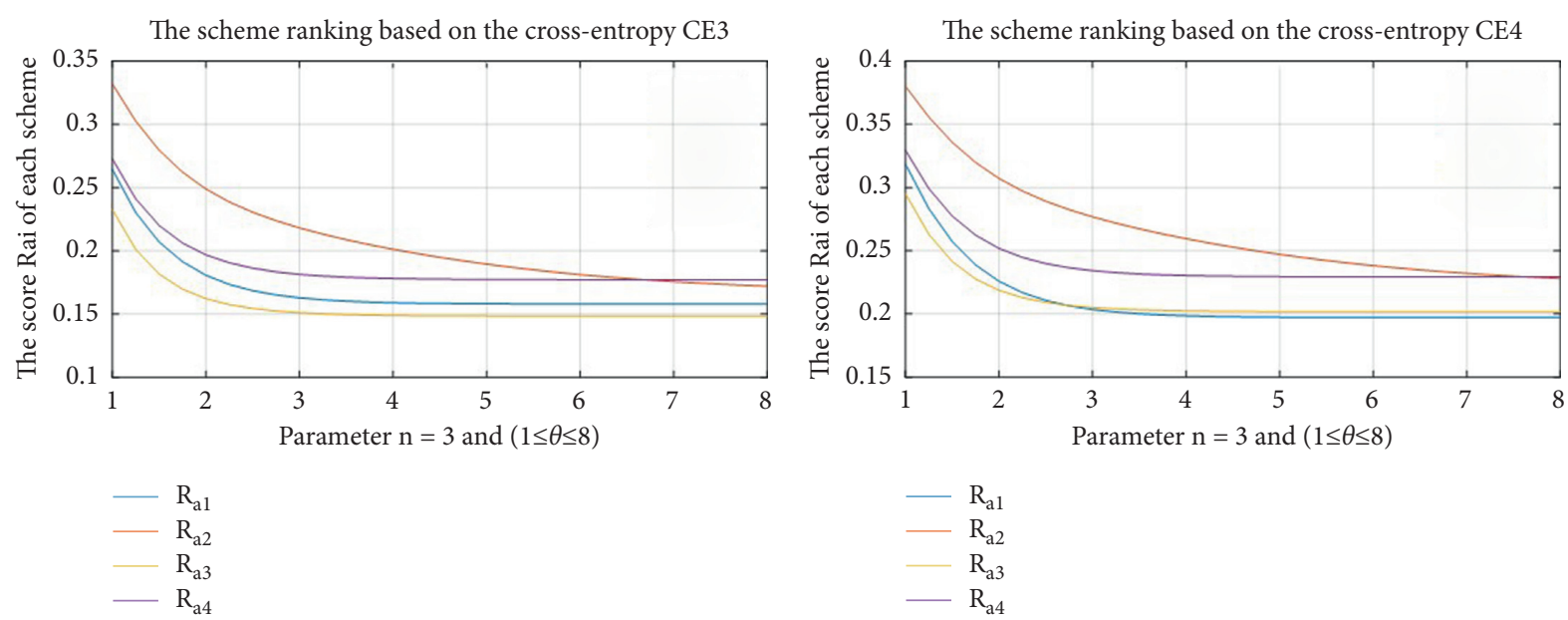

FIGURE 12: Ranking score of each alternative based on parameters $n=3,1 \leq \theta \leq 8$. 

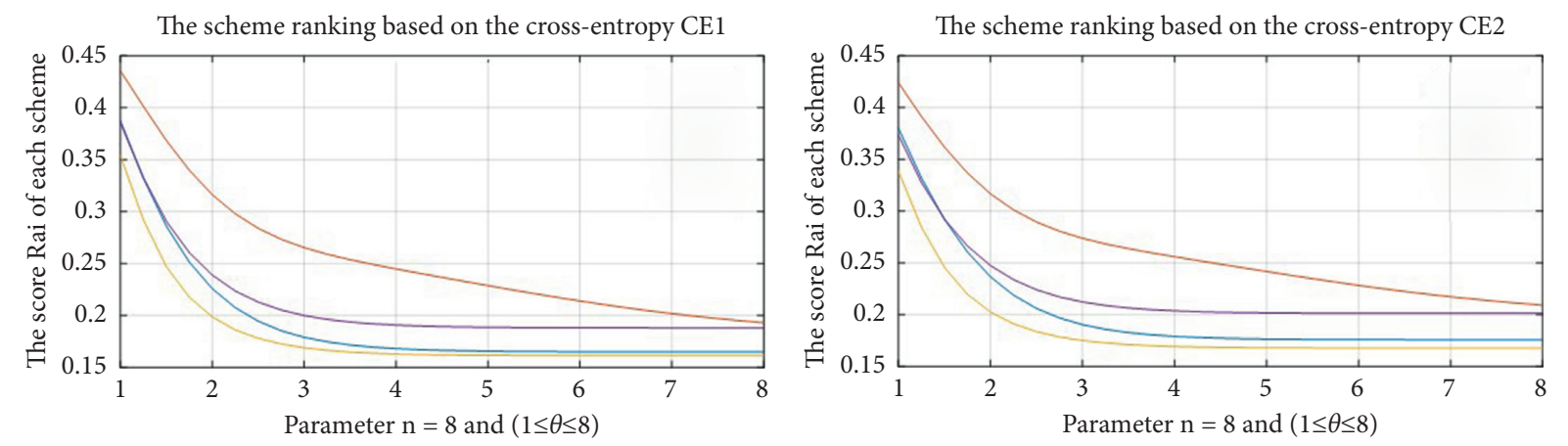

$$
\begin{array}{r}
-\mathrm{R}_{\mathrm{a} 1} \\
-\mathrm{R}_{\mathrm{a} 2} \\
-\mathrm{R}_{\mathrm{a} 3} \\
-\mathrm{R}_{\mathrm{a} 4}
\end{array}
$$

$$
\begin{array}{r}
-\mathrm{R}_{\mathrm{a} 1} \\
-\mathrm{R}_{\mathrm{a} 2} \\
-\mathrm{R}_{\mathrm{a} 3} \\
-\mathrm{R}_{\mathrm{a} 4}
\end{array}
$$
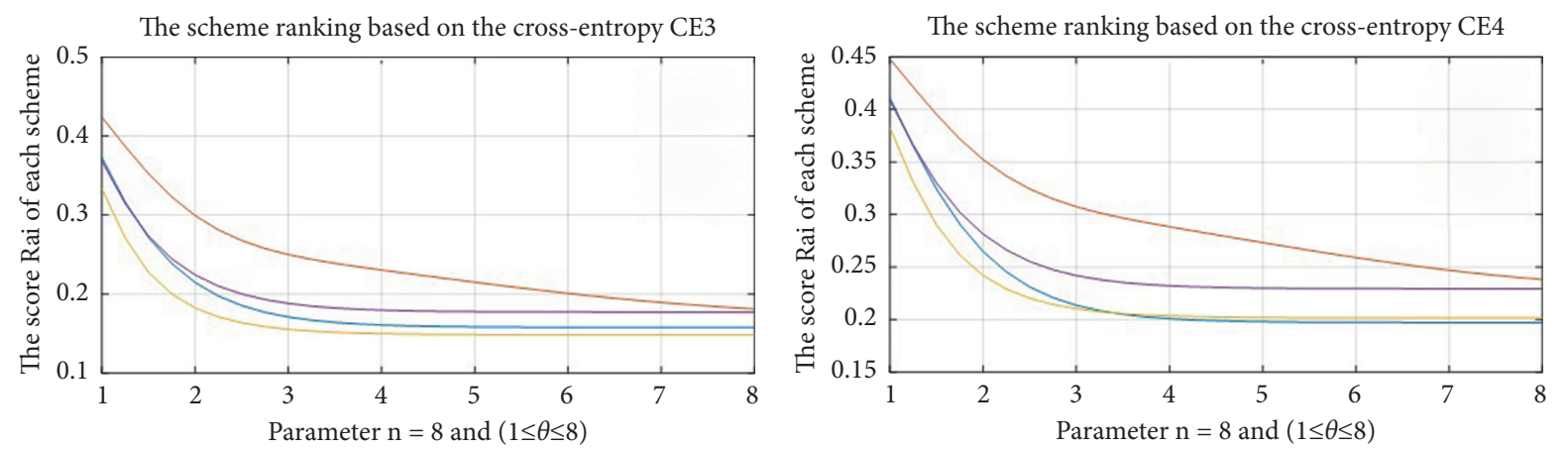

$$
\begin{array}{r}
-\mathrm{R}_{\mathrm{a} 1} \\
-\mathrm{R}_{\mathrm{a} 2} \\
-\mathrm{R}_{\mathrm{a} 3} \\
-\mathrm{R}_{\mathrm{a} 4}
\end{array}
$$$$
\begin{array}{r}
-\mathrm{R}_{\mathrm{a} 1} \\
-\mathrm{R}_{\mathrm{a} 2} \\
-\mathrm{R}_{\mathrm{a} 3} \\
-\mathrm{R}_{\mathrm{a} 4}
\end{array}
$$

FIGURE 13: Ranking score of each alternative based on parameters $n=8,1 \leq \theta \leq 8$. 

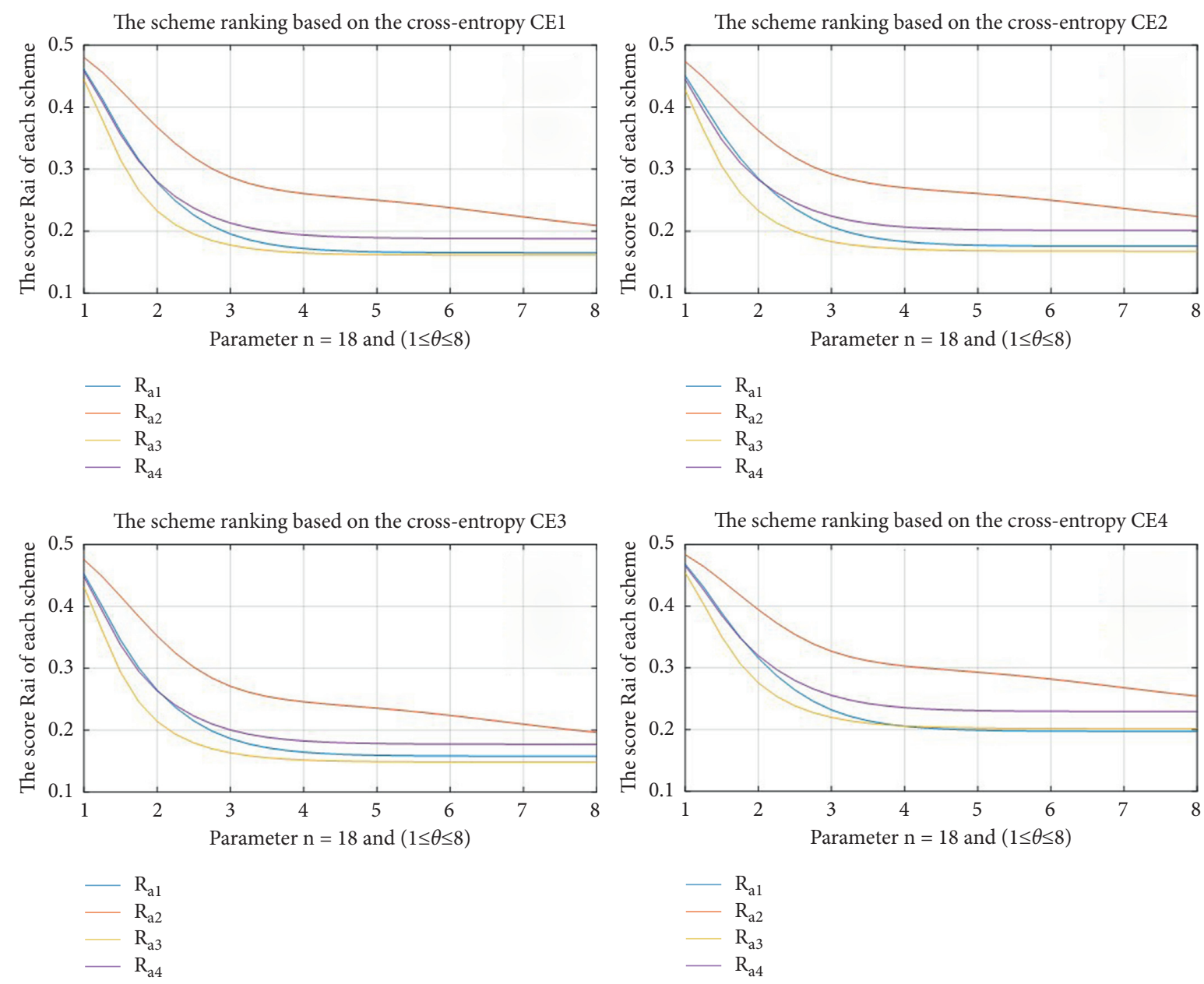

FIGURE 14: Ranking score of each alternative based on parameters $n=18,1 \leq \theta \leq 8$.

TABLE 3: Comparative results obtained by different methods.

\begin{tabular}{lcrr}
\hline Methods & Cross-entropy & Parameter & Rank results \\
\hline Wei [28] & Weighted & None & $a_{4}>a_{2}>a_{3}>a_{1}$ \\
\hline & & $\theta=5, n=-2$ & $a_{4}>a_{3}>a_{1}>a_{2}$ \\
Our methods & Weighted & $\theta=8, n=-2$ & $a_{4}>a_{2}>a_{3}>a_{1}$ \\
& & $\theta=1, n=2$ & $a_{2}>a_{4}>a_{1}>a_{3}$ \\
& & $\theta=10, n=2$ & $a_{4}>a_{3}>a_{1}>a_{2}$ \\
\hline
\end{tabular}

number and linguistic term to HPFLSs is necessary to study in the future. Additionally, according to the requirements of real-life decision-making, the proposed method will be extended to other applications [32-42], or constructing the novel MCDM method with entropy [43-45], evidential reasoning [46], cloud model [47, 48], interactive operators $[49,50]$, power operators [51], TODIM [52], and so forth. 


\section{Data Availability}

The data used to support the findings of this study are included in Table 1 within the article.

\section{Conflicts of Interest}

The authors declare that there are no conflicts of interest regarding the publication of this paper.

\section{Acknowledgments}

This work was supported by the National Natural Science Foundation of China (nos. 71701065) and the Project of Youth and Middle-Aged Innovation Team of Science and Technology of Hubei Province (grant no. T201711).

\section{References}

[1] L. A. Zadeh, "Fuzzy sets," Information Control, vol. 8, no. 3, pp. 338-353, 1965.

[2] X. Sang, X. Liu, and J. Qin, “An analytical solution to fuzzy TOPSIS and its application in personnel selection for knowledge-intensive enterprise," Applied Soft Computing, vol. 30, pp. 190-204, 2015.

[3] M. Dursun and E. E. Karsak, "A fuzzy MCDM approach for personnel selection," Expert Systems with Applications, vol. 37, no. 6 , pp. 4324-4330, 2010.

[4] A. Baležentis, T. Baležentis, and W. K. Brauers, "Personnel selection based on computing with words and fuzzy MULTIMOORA," Expert Systems with Applications, vol. 39, no. 9, pp. 7961-7967, 2012.

[5] H.-T. Lin, "Personnel selection using analytic network process and fuzzy data envelopment analysis approaches," Computers \& Industrial Engineering, vol. 59, no. 4, pp. 937-944, 2010.

[6] G.-S. Liang and M.-J. J. Wang, "Personnel selection using fuzzy MCDM algorithm," European Journal of Operational Research, vol. 78, no. 1, pp. 22-33, 1994.

[7] A. Kelemenis and D. Askounis, "A new TOPSIS-based multicriteria approach to personnel selection," Expert Systems with Applications, vol. 37, no. 7, pp. 4999-5008, 2010.

[8] S.-f. Zhang and S.-y. Liu, "A GRA-based intuitionistic fuzzy multi-criteria group decision making method for personnel selection," Expert Systems with Applications, vol. 38, no. 9, pp. 11401-11405, 2011.

[9] F. E. Boran, S. Genç, and D. Akay, "Personnel selection based on intuitionistic fuzzy sets," Human Factors and Ergonomics in Manufacturing \& Service Industries, vol. 21, no. 5, pp. 493-503, 2011.

[10] H. S. Kilic, A. E. Demirci, and D. Delen, "An integrated decision analysis methodology based on IF-DEMATEL and IF-ELECTRE for personnel selection," Decision Support Systems, vol. 137, Article ID 113360, 2020.

[11] D. Yu, W. Zhang, and Y. Xu, "Group decision making under hesitant fuzzy environment with application to personnel evaluation," Knowledge-Based Systems, vol. 52, pp. 1-10, 2013.

[12] J. Pu, H. Y. Zhang, and J. Q. Wang, “A projection-based TODIM method under multi- valued neutrosophic environments and its application in personnel selection," Neural Computing and Applications, vol. 29, pp. 221-234, 2018.

[13] N. A. Nabeeh, F. Smarandache, M. Abdel-Basset, H. A. ElGhareeb, and A. Aboelfetouh, "An integrated neutrosophic-
TOPSIS approach and its application to personnel selection: a new trend in brain processing and analysis," IEEE Access, vol. 7, pp. 29734-29744, 2019.

[14] X.-H. Wu, J.-Q. Wang, J. J. Peng, and J. Qian, “A novel group decision-making method with probability hesitant interval neutrosophic set and its application in middle-level manager selection," International Journal for Uncertainty Quantification, vol. 8, no. 4, pp. 291-319, 2018.

[15] X.-H. Wu and J.-Q. Wang, "Cross-entropy measures of multivalued neutrosophic sets and its application in selecting middle-level manager," International Journal for Uncertainty Quantification, vol. 7, no. 2, pp. 155-176, 2017.

[16] B. C. Cường, "Picture fuzzy sets," Journal of Computer Science \& Cybernetics, vol. 30, no. 4, p. 409, 2015.

[17] C. Tian, J.-j. Peng, S. Zhang, W.-y. Zhang, and J.-q. Wang, "Weighted picture fuzzy aggregation operators and their applications to multi-criteria decision-making problems," Computers \& Industrial Engineering, vol. 137, Article ID 106037, 2019.

[18] J.-j. Peng, J.-q. Wang, and X.-h. Wu, "Extended ELECTRE I method with multi-hesitant fuzzy information," International Journal of Fuzzy Systems, vol. 21, no. 7, pp. 2192-2203, 2019.

[19] L. Wang, X.-k. Wang, J.-j. Peng, and J.-q. Wang, "The differences in hotel selection among various types of travellers: a comparative analysis with a useful bounded rationality behavioural decision support model," Tourism Management, vol. 76, Article ID 103961, 2020.

[20] C. Tian and J. J. Peng, "An integrated picture fuzzy ANPTODIM multi-criteria decision -making approach for tourism attraction recommendation," Technological and Economic Development of Economy, vol. 26, no. 2, pp. 331-354, 2020.

[21] C. Tian, J. J. Peng, W. Y. Zhang, S. Zhang, and J. Wang, "Tourism environmental impact assessment based on improved AHP and picture fuzzy PROMETHEE II methods," Technological and Economic Development of Economy, vol. 26, no. 2, pp. 355-378, 2020.

[22] K. W. Shen, L. Li, and J. Q. Wang, "Circular economy model for recycling waste resources under government participation: a case study in industrial waste water circulation in China," Technological and Economic Development of Economy, vol. 26, no. 1, pp. 21-47, 2020.

[23] L. Yang, X. H. Wu, and J. Qian, “A novel multicriteria group decision-making approach with hesitant picture fuzzy linguistic information," Mathematical Problems in Engineering, vol. 2020, Article ID 6394028, 19 pages, 2020.

[24] F. Herrera and L. Martinez, "A 2-tuple fuzzy linguistic representation model for computing with words," IEEE Transactions on Fuzzy Systems, vol. 8, no. 6, pp. 746-752, 2000.

[25] R.-x. Nie, J.-q. Wang, and L. Li, “A shareholder voting method for proxy advisory firm selection based on 2-tuple linguistic picture preference relation," Applied Soft Computing, vol. 60, pp. 520-539, 2017.

[26] S. Kullback, Information Theory and Statistics, Wiley, New York, NY, USA, 1959.

[27] X.-G. Shang and W.-S. Jiang, "A note on fuzzy information measures," Pattern Recognition Letters, vol. 18, no. 5, pp. 425-432, 1997.

[28] G. Wei, "Picture fuzzy cross-entropy for multiple attribute decision making problems," Journal of Business Economics and Management, vol. 17, no. 4, pp. 491-502, 2016.

[29] Z.-p. Tian, H.-y. Zhang, J. Wang, J.-q. Wang, and X.-h. Chen, "Multi-criteria decision-making method based on a cross- 
entropy with interval neutrosophic sets," International Journal of Systems Science, vol. 47, no. 15, pp. 3598-3608, 2016.

[30] M. Zeleny, Multiple-Criteria Decision Making, McGraw-Hill, New York, NY, USA, 1982.

[31] Z. Xu, "A deviation-based approach to intuitionistic fuzzy multiple attribute group decision making," Group Decision and Negotiation, vol. 19, no. 1, pp. 57-76, 2010.

[32] Y. Yang, J. Hu, Y. Liu, and X. Chen, "Alternative selection of end-of-life vehicle management in China: a group decisionmaking approach based on picture hesitant fuzzy measurements," Journal of Cleaner Production, vol. 206, pp. 631-645, 2019.

[33] J. Ye, J. Song, S. G. Du, and R. Yong, "Weighted aggregation operators of fuzzy credibility numbers and their decisionmaking approach for slope design schemes," Computational and Applied Mathematics, vol. 40, p. 155, 2021.

[34] J. Ye, S. Du, R. Yong, and F. Zhang, "Arccosine and arctangent similarity measures of refined simplified neutrosophic indeterminate sets and their multicriteria decision-making method," Journal of Intelligent \& Fuzzy Systems, vol. 40, no. 5, pp. 9159-9171, 2021.

[35] J. Wang, J.-q. Wang, Z.-p. Tian, and D.-y. Zhao, "A multihesitant fuzzy linguistic multicriteria decision-making approach for logistics outsourcing with incomplete weight information," International Transactions in Operational Research, vol. 25, no. 3, pp. 831-856, 2018.

[36] X.-h. Wu, J.-q. Wang, J.-j. Peng, and X.-h. Chen, "Crossentropy and prioritized aggregation operator with simplified neutrosophic sets and their application in multi-criteria decision-making problems," International Journal of Fuzzy Systems, vol. 18, no. 6, pp. 1104-1116, 2016.

[37] Z. Zhang, J. Gao, Y. Gao, and W. Yu, "Two-sided matching decision making with multi-granular hesitant fuzzy linguistic term sets and incomplete criteria weight information," Expert Systems with Applications, vol. 168, Article ID $114311,2021$.

[38] H. Seiti, A. Hafezalkotob, and E. Herrera-Viedma, "A novel linguistic approach for multi-granular information fusion and decision-making using risk-based linguistic D numbers," Information Sciences, vol. 530, pp. 43-65, 2020.

[39] P. Liu and L. Rong, "Multiple attribute group decisionmaking approach based on multi-granular unbalanced hesitant fuzzy linguistic information," International Journal of Fuzzy Systems, vol. 22, no. 2, pp. 604-618, 2020.

[40] Z. Zhang, Z. Li, and Y. Gao, "Consensus reaching for group decision making with multi-granular unbalanced linguistic information: a bounded confidence and minimum adjustment-based approach," Information Fusion, vol. 74, pp. 96110, 2021.

[41] F. Teng, P. Liu, and X. Liang, "Unbalanced probabilistic linguistic decision-making method for multi-attribute group decision-making problems with heterogeneous relationships and incomplete information," Artificial Intelligence Review, vol. 54, no. 5, pp. 3431-3471, 2021.

[42] P. Liu and W. Liu, "Multiple-criteria decision making method based on the scaled prioritized operators with unbalanced linguistic information," Artificial Intelligence Review, vol. 53, no. 7, pp. 4967-4991, 2020.

[43] S.-P. Wan, Z. Jin, and J.-Y. Dong, "Pythagorean fuzzy mathematical programming method for multi-attribute group decision making with Pythagorean fuzzy truth degrees," Knowledge and Information Systems, vol. 55, no. 2, pp. 437-466, 2018.
[44] G.-1. Xu, S.-P. Wan, and J.-Y. Dong, "An entropy-based method for probabilistic linguistic group decision making and its application of selecting car sharing platforms," Informatica, vol. 31, no. 3, pp. 621-658, 2020.

[45] S.-p. Wan, W.-c. Zou, L.-g. Zhong, and J.-y. Dong, "Some new information measures for hesitant fuzzy PROMETHEE method and application to green supplier selection," Soft Computing, vol. 24, no. 12, pp. 9179-9203, 2020.

[46] H. Zhou, J.-q. Wang, H.-y. Zhang, and X.-h. Chen, "Linguistic hesitant fuzzy multi-criteria decision-making method based on evidential reasoning," International Journal of Systems Science, vol. 47, no. 2, pp. 314-327, 2016.

[47] J.-Q. Wang, P. Wang, J. Wang, H.-Y. Zhang, and X.-H. Chen, "Atanassov's interval-valued intuitionistic linguistic multicriteria group decision-making method based on the trapezium cloud model," IEEE Transactions on Fuzzy Systems, vol. 23, no. 3, pp. 542-554, 2015.

[48] J.-q. Wang, J.-j. Peng, H.-y. Zhang, T. Liu, and X.-h. Chen, "An uncertain linguistic multi-criteria group decision-making method based on a cloud model," Group Decision and Negotiation, vol. 24, no. 1, pp. 171-192, 2015.

[49] L. Wang and H. Garg, "Algorithm for multiple attribute decision-making with interactive archimedean norm operations under pythagorean fuzzy uncertainty," International Journal of Computational Intelligence Systems, vol. 14, no. 1, pp. 503-527, 2021.

[50] L. Wang and N. Li, "Pythagorean fuzzy interaction power Bonferroni mean aggregation operators in multiple attribute decision making," International Journal of Intelligent Systems, vol. 35, no. 1, pp. 150-183, 2020.

[51] L. Wang, Q. Shen, and L. Zhu, "Dual hesitant fuzzy power aggregation operators based on Archimedean $t$-conorm and $t$ norm and their application to multiple attribute group decision making," Applied Soft Computing, vol. 38, pp. 23-50, 2016.

[52] R. X. Nie, Z. P. Tian, C. K. Sang, and J. Wang, "Implementing healthcare service quality enhancement using a cloud-support QFD model integrated with TODIM method and linguistic distribution assessments," Journal of the Operational Research Society, pp. 1-23, 2020. 